\title{
Hierarchical slope limiting in explicit and implicit discontinuous Galerkin methods
}

\author{
Dmitri Kuzmin \\ Applied Mathematics III, University Erlangen-Nuremberg \\ Cauerstr. 11, D-91058, Erlangen, Germany
}

\begin{abstract}
In this paper, we present a collection of algorithmic tools for constraining high-order discontinuous Galerkin (DG) approximations to hyperbolic conservation laws. We begin with a review of hierarchical slope limiting techniques for explicit DG methods. A new interpretation of these techniques leads to an unconditionally stable implicit algorithm for steady-state computations. The implicit global problem for the mean values (coarse scales) has the computational structure of a finite volume method. The constrained derivatives (fine scales) are obtained by solving small local problems. The interscale transfer operators provide a two-way iterative coupling between the solutions to the global and local problems. Another highlight of this paper is a new approach to compatible gradient limiting for the Euler equations of gas dynamics. After limiting the conserved quantities, the gradients of the velocity and energy density are constrained in a consistent manner. Numerical studies confirm the accuracy and robustness of the proposed algorithms.
\end{abstract}

Key words: hyperbolic conservation laws, discontinuous Galerkin methods, variational multiscale methods, slope limiting, constrained optimization

\section{Introduction}

Discontinuous Galerkin (DG) methods [8, 9] belong to the most popular numerical techniques for Computational Fluid Dynamics. Bridging the gap between finite volumes and finite elements, the DG approach is particularly well suited for numerical treatment of hyperbolic conservation laws. The underlying variational formulation is locally conservative, and the discrete

Email address: kuzmin@am.uni-erlangen.de (Dmitri Kuzmin) 
problem can be written in terms of upwind-biased numerical fluxes. The use of slope limiting techniques makes it possible to control the jumps of the DG solution by constraining the high-order components of the shape function or setting them to zero in "troubled" cells [2, 3, 7, 10, 11, 22, 35, 36, 41, 42].

A well-designed slope limiter filters out nonphysical oscillations without degrading the order of accuracy at smooth peaks. In an attempt to satisfy both requirements, monotonicity-preserving limiters are frequently combined with heuristic oscillation detectors. Clearly, the robustness of such a method depends on the underlying criteria for distinguishing between "good" and "bad" discontinuities. It also depends on the choice of free parameters. Moreover, classical slope limiters are tailored for constraining linear reconstructions. Their extension to high-order discontinuous Galerkin approximations is difficult due to the lack of generalized maximum principles.

In this paper, we discuss some improvements and new generalizations of the hierarchical slope limiter proposed in $[24,25]$. In the case of an explicit DG scheme, this limiter constrains the derivatives in the Taylor polynomial form of the shape function so as to eliminate undershoots and overshoots at the vertices of the mesh. A derivative of degree $p \geq 1$ is limited in such a way that the value of the $(p-1)$-st derivative at each vertex is bounded by the cell averages in elements containing this vertex. Since derivatives of degree $p$ possess higher regularity than those of degree $q>p$, they are limited by at most the same amount. This hierarchical approach to slope limiting preserves smooth peaks without resorting to troubled cell markers.

The above limiting strategy has a lot in common with other methods proposed in the literature. The use of Taylor basis functions was inspired by the work of Luo et al. [32], Michalak and Ollivier-Gooch [35], and Yang and Wang [42]. The bounds for the solution values at the vertices of the mesh are defined as in the paper by Hoteit et al. [19] and enforced using a modification of the Barth-Jespersen limiter [2]. As shown by Aizinger [1], the resulting algorithm can be interpreted as a local optimization problem. It is simpler than the one formulated in [19] since mass conservation is guaranteed by the properties of the Taylor basis and does not need to be imposed as an additional constraint. The far-reaching idea of limiting higher-order terms in a hierarchical manner is due to Krivodonova [22] who developed a generalized moment limiter for a tensor-product basis of Legendre polynomials. Our vertex-based hierarchical limiter [24] is based on the same design philosophy but is applicable to arbitrary meshes and basis functions. Representation in terms of the Taylor basis is required for limiting purposes only [36]. 
In the original paper [24], we used an explicit Runge-Kutta time-stepping method and neglected the off-diagonal part of the Taylor mass matrix to avoid spurious distortions due to implicit coupling between the unconstrained derivatives. This mass lumping strategy is conservative but results in a loss of accuracy, especially in higher-order approximations. As a remedy, we developed a predictor-corrector algorithm that eliminates the need for mass lumping using the vertex-based limiter to pre-constrain the discretized time derivatives [25]. This strategy was found to eliminate the side effects caused by the non-orthogonality of the Taylor basis on triangular meshes.

In what follows, we extend the methodology developed in $[24,25]$ to implicit time discretizations and nonlinear systems of conservation laws. The design of an unconditionally stable implicit algorithm is based on an analogy with variational multiscale (VMS) methods. As shown by Bochev et al. $[5,20]$, the multiscale nature of DG approximations provides useful insights for the design of efficient algorithms. In particular, an additive decomposition of the finite element space into continuous coarse scales and discontinuous fine scales leads to a DG scheme with the computational structure of a continuous Galerkin method [5]. In this paper, we split each shape function into the cell mean value and higher-order terms. The result is a DG scheme in which the coarse-scale problem has the computational structure of a finite volume method. The fine-scale components (derivatives) are filtered using slope limiting or inequality-constrained optimization. The global coarse-scale problem and local fine-scale problems are solved sequentially using the constrained solution from the previous iteration to calculate the fluxes. In contrast to the full $p$-multigrid algorithm [31, 33], all fine-scale components associated with the same element are updated simultaneously.

Other ideas to be discussed include the use of boundary conditions in the definition of upper and lower bounds for the slope limiter, derivative recovery via reconstruction techniques, and limiter-based smoothness indicators for $h p$-adaptivity in finite element codes. Last but not least, we extend the vertex-based slope limiter [24] to the compressible Euler equations and introduce a compatible gradient limiting strategy for coupled variables. The proposed algorithm makes it possible to constrain the velocity and energy density using the product rule to calculate the gradients. Numerical studies are performed for standard two-dimensional test problems. The two-scale iterative solver is shown to be unconditionally stable. The results for the Euler equations indicate that compatible gradient limiting eliminates the ripples that arise when the conserved quantities are limited independently. 


\section{Global and local problems}

The first model problem that we will use for presentation of the constrained DG method in this paper is the linear convection equation

$$
\frac{\partial u}{\partial t}+\nabla \cdot(\mathbf{v} u)=0 \quad \text { in } \Omega
$$

where $\Omega$ is a bounded domain, $u(\mathbf{x}, t)$ is a conserved scalar quantity, and $\mathbf{v}(\mathbf{x}, t)$ is a (continuous) velocity field. The initial condition is given by

$$
u(\cdot, 0)=u_{0} \quad \text { in } \Omega .
$$

The hyperbolic inlet is defined as $\Gamma_{\text {in }}=\{\mathbf{x} \in \Gamma \mid \mathbf{v}(\mathbf{x}) \cdot \mathbf{n}(\mathbf{x})<0\}$, where $\mathbf{n}$ denotes the unit outward normal to the boundary $\Gamma=\partial \Omega$. The Dirichlet boundary condition $u=u_{\text {in }}$ on $\Gamma_{\text {in }}$ is imposed in a weak sense as follows:

$$
u \mathbf{v} \cdot \mathbf{n}=u_{\text {in }} \mathbf{v} \cdot \mathbf{n} \quad \text { on } \Gamma_{\text {in }} .
$$

This formula defines the incoming convective flux. In accordance with the hyperbolic PDE theory, no boundary conditions are prescribed on $\Gamma \backslash \Gamma_{i n}$.

Remark 1. We are also interested in steady state solutions to the above problem, i.e., in solutions to (1) that are obtained in the limit $\frac{\partial u}{\partial t} \rightarrow 0$.

To formulate the discontinuous Galerkin method, we consider an arbitrary element $K \in \mathcal{T}_{h}$ of a (possibly unstructured) computational mesh $\mathcal{T}_{h}$. Multiplying (1) by a suitable test function $w$, integrating over $K$, and using Green's formula, one obtains the local variational formulation

$$
\int_{K}\left(w \frac{\partial u}{\partial t}-\nabla w \cdot \mathbf{v} u\right) \mathrm{d} \mathbf{x}+\int_{\partial K} w \hat{u} \mathbf{v} \cdot \mathbf{n} \mathrm{d} s=0, \quad \forall w \in V,
$$

where $V$ is the space of admissible test functions and $\hat{u}$ is the upwind-sided trace of the (generally discontinuous) function $u: \Omega \rightarrow \mathbb{R}$.

The inflow and outflow boundaries of element $K$ are defined by

$$
\begin{gathered}
\partial K_{\mathrm{in}}=\{\mathbf{x} \in \partial K \mid \mathbf{v}(\mathbf{x}) \cdot \mathbf{n}(\mathbf{x})<0\} \\
\partial K_{\text {out }}=\{\mathbf{x} \in \partial K \mid \mathbf{v}(\mathbf{x}) \cdot \mathbf{n}(\mathbf{x})>0\} .
\end{gathered}
$$

By virtue of $(3)$, we have $\hat{u}=u_{\text {in }}$ on $\partial K_{\text {in }} \cap \Gamma_{\text {in }}$. On the interface between $K$ and another element $K^{\prime} \in \mathcal{T}_{h}$, the traces are given by the one-sided limits

$$
u^{ \pm}(\mathbf{x}, t)=\lim _{\epsilon \rightarrow \pm 0} u(\mathbf{x}+\epsilon \mathbf{n}, t), \quad \mathbf{x} \in \partial K .
$$


Selecting the upwind-sided value, we define the convective fluxes using

$$
\hat{u}(\mathbf{x}, t)= \begin{cases}u^{+}(\mathbf{x}, t) & \text { if } \mathbf{x} \in \partial K_{\mathrm{in}} \backslash \Gamma_{\mathrm{in}}, \\ u_{\mathrm{in}}(\mathbf{x}, t) & \text { if } \mathbf{x} \in \partial K_{\mathrm{in}} \cap \Gamma_{\mathrm{in}}, \\ u^{-}(\mathbf{x}, t) & \text { otherwise. }\end{cases}
$$

The generic form of the local problem associated with element $K$ reads

$$
\left(w, \frac{\partial u}{\partial t}\right)_{K}+a_{K}(w, u)=b_{K}(w), \quad \forall w \in V,
$$

where

$$
\begin{aligned}
& a_{K}(w, u)=\int_{\partial K_{\mathrm{out}}} w u \mathbf{v} \cdot \mathbf{n} \mathrm{d} s-\int_{K} \nabla w \cdot \mathbf{v} u \mathrm{~d} \mathbf{x}, \\
& b_{K}(w)=-\int_{\partial K_{\mathrm{in}}} w \hat{u} \mathbf{v} \cdot \mathbf{n} \mathrm{d} s, \quad\left(w, \frac{\partial u}{\partial t}\right)_{K}=\int_{K} w \frac{\partial u}{\partial t} \mathrm{~d} \mathbf{x} .
\end{aligned}
$$

The global variational formulation is obtained by adding together all local problems. Consider a pair of neighbor elements $K, K^{\prime} \in \mathcal{T}_{h}$ that have a common edge/face $S=\partial K \cap \partial K^{\prime}$ with the unit normal $\mathbf{n}$ pointing from $K$ into $K^{\prime}$. The sum of the corresponding surface integrals can be written as

$$
\int_{S} w^{-} \hat{u} \mathbf{v} \cdot \mathbf{n} \mathrm{d} s-\int_{S} w^{+} \hat{u} \mathbf{v} \cdot \mathbf{n} \mathrm{d} s=\int_{S} \llbracket w \rrbracket \hat{u} \mathbf{v} \cdot \mathbf{n} \mathrm{d} s,
$$

where $\llbracket w \rrbracket:=w^{-}-w^{+}$is the jump of $w$ across $S$. Thus the sum of integrals over the internal boundaries of all elements $K \in \mathcal{T}_{h}$ equals the sum of jump terms over $S \in \mathcal{S}_{h}$, where $\mathcal{S}_{h}$ denotes the set of all internal edges/faces.

In light of the above, the global semi-discrete problem is given by

$$
\left(w, \frac{\partial u}{\partial t}\right)+a(w, u)=b(w), \quad \forall w \in V
$$

where

$$
\begin{aligned}
& a(w, u)=\sum_{S \in \mathcal{S}_{h}} \int_{S} \llbracket w \rrbracket \hat{u} \mathbf{v} \cdot \mathbf{n} \mathrm{d} s+\int_{\Gamma \backslash \Gamma_{\mathrm{in}}} w u \mathbf{v} \cdot \mathbf{n} \mathrm{d} s-\sum_{K \in \mathcal{T}_{h}} \int_{K} \nabla w \cdot \mathbf{v} u \mathrm{~d} \mathbf{x}, \\
& b(w)=-\int_{\Gamma_{\mathrm{in}}} w u_{\mathrm{in}} \mathbf{v} \cdot \mathbf{n} \mathrm{d} s, \quad\left(w, \frac{\partial u}{\partial t}\right)=\sum_{K \in \mathcal{T}_{h}} \int_{K} w \frac{\partial u}{\partial t} \mathrm{~d} \mathbf{x} .
\end{aligned}
$$

Remark 2. Setting $w \equiv 1$ in the local or global problem, one obtains the integral form of the conservation law for $K$ and $\Omega$, respectively. This proves that the variational formulation is locally and globally conservative. 


\section{DG space discretization}

Leaving the time variable continuous for the time being, we discretize (6) in space using the finite element method. The weak solution $u$ is approximated by a linear combination of piecewise-polynomial basis functions $\left\{\varphi_{j}\right\}$ spanning a finite-dimensional space $V_{h}$. The restriction of the numerical solution $u_{h}$ to an element $K \in \mathcal{T}_{h}$ is a continuous shape function given by

$$
u_{h}(\mathbf{x}, t)=\sum_{j} u_{j}(t) \varphi_{j}(\mathbf{x}), \quad \mathbf{x} \in K
$$

Restricting (6) to functions from $V_{h}$, we look for $u_{h} \in V_{h}$ such that

$$
\left(w_{h}, \frac{\partial u_{h}}{\partial t}\right)_{K}+a_{K}\left(w_{h}, u_{h}\right)=b_{K}\left(w_{h}\right), \quad \forall w_{h} \in V_{h} .
$$

By (12), this variational problem is equivalent to the linear system

$$
\sum_{j} \frac{\mathrm{d} u_{i}}{\mathrm{~d} t}\left(\varphi_{i}, \varphi_{j}\right)_{K}+\sum_{j} u_{j} a_{K}\left(\varphi_{i}, \varphi_{i}\right)=b_{K}\left(\varphi_{i}\right), \quad \forall i
$$

The matrix form of the so-defined semi-discrete local problem reads

$$
M_{K} \frac{\mathrm{d} u_{K}}{\mathrm{~d} t}+A_{K} u_{K}=f_{K}
$$

where $M_{K}=\left\{m_{i j}\right\}$ is the element mass matrix, $A_{K}=\left\{a_{i j}\right\}$ is the discrete convection operator (including the outgoing fluxes), and $f_{K}=\left\{f_{i}\right\}$ is the vector of incoming fluxes. By (14), the coefficients are given by

$$
\begin{gathered}
m_{i j}=\left(\varphi_{i}, \varphi_{j}\right)_{K}, \\
a_{i j}=a_{K}\left(\varphi_{i}, \varphi_{i}\right), \\
f_{i}=b_{K}\left(\varphi_{i}\right) .
\end{gathered}
$$

The global system can be assembled from element contributions which are coupled through the surface integrals in the coefficients $a_{i j}$ and $f_{i}$. Note that there is no need for global matrix assembly if the time integration is explicit or the algebraic systems are solved using a matrix-free iterative method. 


\section{Taylor basis functions}

In a discontinuous Galerkin method of degree $p \geq 0$, the shape function $\left.u_{h}\right|_{K}$ is given by (12), where the number of basis functions depends on $p$. Following Luo et al. [32], we restrict our discussion to quadratic polynomials $\left.u_{h}\right|_{K} \in P_{2}(K)$ and consider the two-dimensional Taylor expansion

$$
\begin{aligned}
u_{h}(x, y) & =u_{c}+\left.\frac{\partial u}{\partial x}\right|_{c}\left(x-x_{c}\right)+\left.\frac{\partial u}{\partial y}\right|_{c}\left(y-y_{c}\right)+\left.\frac{\partial^{2} u}{\partial x^{2}}\right|_{c} \frac{\left(x-x_{c}\right)^{2}}{2} \\
& +\left.\frac{\partial^{2} u}{\partial y^{2}}\right|_{c} \frac{\left(y-y_{c}\right)^{2}}{2}+\left.\frac{\partial^{2} u}{\partial x \partial y}\right|_{c}\left(x-x_{c}\right)\left(y-y_{c}\right)
\end{aligned}
$$

about the centroid $\left(x_{c}, y_{c}\right)$ of $K \in \mathcal{T}_{h}$. Introducing the volume averages

$$
\bar{u}_{K}=\frac{1}{|K|} \int_{K} u_{h} \mathrm{~d} \mathbf{x},\left.\quad \overline{x^{n} y^{m}}\right|_{K}=\frac{1}{|K|} \int_{K} x^{n} y^{m} \mathrm{~d} \mathbf{x}
$$

the quadratic function $u_{h}$ can be expressed in the equivalent form $[32,35,42]$

$$
\begin{aligned}
u_{h}(x, y) & =\bar{u}_{K}+\left.\frac{\partial u}{\partial x}\right|_{c}\left(x-x_{c}\right)+\left.\frac{\partial u}{\partial y}\right|_{c}\left(y-y_{c}\right) \\
& +\left.\frac{\partial^{2} u}{\partial x^{2}}\right|_{c}\left[\frac{\left(x-x_{c}\right)^{2}}{2}-\frac{\overline{\left(x-x_{c}\right)^{2}}}{2}\right]+\left.\frac{\partial^{2} u}{\partial y^{2}}\right|_{c}\left[\frac{\left(y-y_{c}\right)^{2}}{2}-\frac{\overline{\left(y-y_{c}\right)^{2}}}{2}\right] \\
& +\left.\frac{\partial^{2} u}{\partial x \partial y}\right|_{c}\left[\left(x-x_{c}\right)\left(y-y_{c}\right)-\overline{\left(x-x_{c}\right)\left(y-y_{c}\right)}\right] .
\end{aligned}
$$

This representation has led Luo et al. [32] to consider the local Taylor basis

$$
\begin{aligned}
& \varphi_{1}=1, \quad \varphi_{2}=\frac{x-x_{c}}{\Delta x}, \quad \varphi_{3}=\frac{y-y_{c}}{\Delta y}, \quad \varphi_{4}=\frac{\left(x-x_{c}\right)^{2}}{2 \Delta x^{2}}-\frac{\overline{\left(x-x_{c}\right)^{2}}}{2 \Delta x^{2}}, \\
& \varphi_{5}=\frac{\left(y-y_{c}\right)^{2}}{2 \Delta y^{2}}-\frac{\overline{\left(y-y_{c}\right)^{2}}}{2 \Delta y^{2}}, \quad \varphi_{6}=\frac{\left(x-x_{c}\right)\left(y-y_{c}\right)-\overline{\left(x-x_{c}\right)\left(y-y_{c}\right)}}{\Delta x \Delta y} .
\end{aligned}
$$

The scaling by $\Delta x=\left(x_{\max }-x_{\min }\right) / 2$ and $\Delta y=\left(y_{\max }-y_{\min }\right) / 2$ is required to obtain a well-conditioned system [32]. The normalized degrees of freedom are proportional to the cell mean value $\bar{u}_{K}$ and derivatives of $u_{h}$ at $\left(x_{c}, y_{c}\right)$

$$
\begin{aligned}
u_{h}(x, y) & =\bar{u}_{K} \varphi_{1}+\left(\left.\frac{\partial u}{\partial x}\right|_{c} \Delta x\right) \varphi_{2}+\left(\left.\frac{\partial u}{\partial y}\right|_{c} \Delta y\right) \varphi_{3}+\left(\left.\frac{\partial^{2} u}{\partial x^{2}}\right|_{c} \Delta x^{2}\right) \varphi_{4} \\
& +\left(\left.\frac{\partial^{2} u}{\partial y^{2}}\right|_{c} \Delta y^{2}\right) \varphi_{5}+\left(\left.\frac{\partial^{2} u}{\partial x \partial y}\right|_{c} \Delta x \Delta y\right) \varphi_{6} .
\end{aligned}
$$


On a uniform mesh of rectangular elements, the Taylor basis (21) is orthogonal [10] and yields a diagonal mass matrix $M_{K}$. In general, we have

$$
m_{11}=|K|, \quad m_{1 j}=m_{j 1}=0, \quad 2 \leq j \leq 6 .
$$

However, higher-order Taylor basis functions are non-orthogonal to one another on triangular meshes and on general quadrilateral meshes. A nondiagonal mass matrix $M_{K}$ may be 'lumped' by setting all off-diagonal entries equal to zero. In contrast to the case of a typical Lagrange basis, this modification is conservative but mass lumping degrades phase accuracy and should be avoided whenever possible (see the numerical examples below).

\section{Multiscale decomposition}

The above representation of $u_{h}$ in terms of Taylor basis functions makes it possible to limit the gradients and second derivatives in a conservative manner. Moreover, the Taylor basis defines the two-scale decomposition

$$
u_{h}=\bar{u}_{h}+u_{h}^{\prime}
$$

where the coarse-scale component $\bar{u}_{h}$ is the mean value of $u_{h}$ over $K \in \mathcal{T}_{h}$ and $u_{h}^{\prime}=u_{h}-\bar{u}_{h}$ is the contribution of linear and quadratic terms in (20).

The high-order part admits a further additive decomposition into resolved fine scales $\bar{u}_{h}^{\prime}$ and unresolved fine scales $\bar{u}_{h}^{\prime \prime}=u_{h}^{\prime}-\bar{u}_{h}^{\prime}$. This gives

$$
u_{h}^{\prime}=\bar{u}_{h}^{\prime}+u_{h}^{\prime \prime} \text {. }
$$

Since unresolvable fluctuations $u_{h}^{\prime \prime}$ tend to produce nonphysical oscillations, they should be removed by imposing certain constraints on the fine-scale problem. The filtered component $\bar{u}_{h}^{\prime}$ can be extracted using hierarchical slope limiting $[22,24,42]$ of by solving a constrained minimization problem $[1,19,37]$. Both approaches are discussed in detail in this paper.

The Taylor basis functions associated with $\bar{u}_{h}$ and $u_{h}^{\prime}$ are given by $\left\{\varphi_{1}\right\}$ and $\left\{\varphi_{2}, \ldots, \varphi_{6}\right\}$, respectively. The space of test functions admits a similar decomposition. Thus, the local problem can be split into two subproblems

$$
\begin{aligned}
& \left(\bar{w}_{h}, \frac{\mathrm{d} \bar{u}_{h}}{\mathrm{~d} t}+\frac{\partial u_{h}^{\prime}}{\partial t}\right)_{K}+a_{K}\left(\bar{w}_{h}, \bar{u}_{h}+u_{h}^{\prime}\right)=b_{K}\left(\bar{w}_{h}\right), \quad \forall \bar{w}_{h} \in \bar{V}_{h}, \\
& \left(w_{h}^{\prime}, \frac{\mathrm{d} \bar{u}_{h}}{\mathrm{~d} t}+\frac{\partial u_{h}^{\prime}}{\partial t}\right)_{K}+a_{K}\left(w_{h}^{\prime}, \bar{u}_{h}+u_{h}^{\prime}\right)=b_{K}\left(w_{h}^{\prime}\right), \quad \forall w_{h}^{\prime} \in V_{h}^{\prime},
\end{aligned}
$$


where $\bar{V}_{h}=\operatorname{span}\left\{\varphi_{1}\right\}$ and $V_{h}^{\prime}=\operatorname{span}\left\{\varphi_{2}, \ldots, \varphi_{6}\right\}$ are the local finite element spaces associated with the coarse and fine scales, respectively.

By definition of the Taylor basis, the first subproblem reduces to a finite volume method. Plugging $\bar{w}_{h}=\varphi_{1}$ into (26) and rearranging, we obtain

$$
|K| \frac{\mathrm{d} \bar{u}_{K}}{\mathrm{~d} t}+\bar{u}_{K} \int_{\partial K_{\text {out }}} \mathbf{v} \cdot \mathbf{n} \mathrm{d} s=-\int_{\partial K_{\text {in }}} \hat{u}_{h} \mathbf{v} \cdot \mathbf{n} \mathrm{d} s-\int_{\partial K_{\text {out }}} u_{h}^{\prime} \mathbf{v} \cdot \mathbf{n} \mathrm{d} s .
$$

The first integral in the right-hand side defines the inflow boundary conditions for $K$. By definition (5), the value of this integral not depend on the solution inside $K$. The influence of fine-scale fluctuations $u_{h}^{\prime}$ inside $K$ is taken into account by the second integral in the right-hand side.

Conversely, the coarse-scale solution $\bar{u}_{h}$ influences the evolution of the fine-scale component $u_{h}^{\prime}$ by changing the magnitude of the convective flux. Using the test function $w_{h}^{\prime}=\varphi_{i}, i=2, \ldots, 6$, we can write (27) thus:

$$
\begin{gathered}
\int_{K}\left(\varphi_{i} \frac{\partial u_{h}^{\prime}}{\partial t}-\nabla \varphi_{i} \cdot \mathbf{v} u_{h}^{\prime}\right) \mathrm{d} \mathbf{x}+\int_{\partial K_{\text {out }}} \varphi_{i} u_{h}^{\prime} \mathbf{v} \cdot \mathbf{n} \mathrm{d} s=-\int_{\partial K_{\mathrm{in}}} \varphi_{i} \hat{u}_{h} \mathbf{v} \cdot \mathbf{n} \mathrm{d} s \\
+\bar{u}_{K} \int_{K} \nabla \varphi_{i} \cdot \mathbf{v} \mathrm{d} \mathbf{x}-\bar{u}_{K} \int_{\partial K_{\text {out }}} \varphi_{i} \mathbf{v} \cdot \mathbf{n} \mathrm{d} s
\end{gathered}
$$

The last two integrals in the right-hand side of this formula describe the response of the fine-scale problem to the coarse-scale solution inside $K$.

In explicit DG schemes, the coarse and fine scales can be updated simultaneously by solving the fully discrete version of (15). However, the separation of coarse and fine scales turns out to be very handy when it comes to the design of iterative solvers for implicit DG schemes (see Section 10).

Since the unconstrained solution to (29) may exhibit spurious undershoots and overshoots, it requires certain postprocessing to extract the filtered finescale fluctuation $\bar{u}_{h}^{\prime}$. In the following sections, we describe the constraints to be imposed and present some practical tools for enforcing them.

\section{Inequality constraints}

Following Krivodonova [21], we constrain the fine-scale components by limiting the derivatives in a hierarchical manner. Given a two-dimensional multiindex $p=\left(p_{1}, p_{2}\right)$, the Taylor degree of freedom associated with the partial derivative $\frac{\partial^{|p|} u}{\partial x^{p_{1}} \partial y^{p_{2}}}$ of degree $|p|=p_{1}+p_{2}>0$ is denoted by

$$
u_{K}^{\left(p_{1}, p_{2}\right)}=\left.\frac{\partial^{|p|} u}{\partial x^{p_{1}} \partial y^{p_{2}}}\right|_{c} \Delta x^{p_{1}} \Delta y^{p_{2}}
$$


whereas $u_{K}^{(0,0)}:=\bar{u}_{K}$ denotes the cell-averaged value of the shape function.

The first-order Taylor polynomial associated with $u_{K}^{\left(p_{1}, p_{2}\right)}$ is given by

$$
T_{K}^{\left(p_{1}, p_{2}\right)}(x, y)=u_{K}^{\left(p_{1}, p_{2}\right)}+u_{K}^{\left(p_{1}+1, p_{2}\right)} \frac{x-x_{c}}{\Delta x}+u_{K}^{\left(p_{1}, p_{2}+1\right)} \frac{y-y_{c}}{\Delta y} .
$$

The linear polynomial $T_{K}^{\left(p_{1}, p_{2}\right)}$ attains its maxima and minima at the vertices of $K$. Thus, the following local maximum principle holds

$$
\min _{i \in \mathcal{V}(K)} T_{K}^{\left(p_{1}, p_{2}\right)}\left(x_{i}, y_{i}\right) \leq T_{K}^{\left(p_{1}, p_{2}\right)}(x, y) \leq \max _{i \in \mathcal{V}(K)} T_{K}^{\left(p_{1}, p_{2}\right)}\left(x_{i}, y_{i}\right)
$$

where $\mathcal{V}(K)$ denotes the set of vertex numbers for element $K \in \mathcal{T}_{h}$.

In the slope-limited DG method [24], inequality constraints of the form

$$
u_{i, \min }^{\left(p_{1}, p_{2}\right)} \leq T_{K}^{\left(p_{1}, p_{2}\right)}\left(x_{i}, y_{i}\right) \leq u_{i, \max }^{\left(p_{1}, p_{2}\right)}, \quad \forall i \in \mathcal{V}(K)
$$

are used to extract the nonoscillatory fine-scale components $[21,24]$. We define the bounds $u_{i, \min }^{\left(p_{1}, p_{2}\right)}$ and $u_{i, \max }^{\left(p_{1}, p_{2}\right)}$ as the maximum and minimum values of $u_{K}^{\left(p_{1}, p_{2}\right)}$ in elements containing the vertex $\left(x_{i}, y_{i}\right)$. That is,

$$
\begin{gathered}
u_{i, \max }^{\left(p_{1}, p_{2}\right)}=\max \left\{u_{K}^{\left(p_{1}, p_{2}\right)} \mid K \in \mathcal{T}_{h}, i \in \mathcal{V}(K)\right\}, \\
u_{i, \min }^{\left(p_{1}, p_{2}\right)}=\min \left\{u_{K}^{\left(p_{1}, p_{2}\right)} \mid K \in \mathcal{T}_{h}, i \in \mathcal{V}(K)\right\} .
\end{gathered}
$$

In practice, the bounds are initialized by a small/large constant and updated in a loop over elements as follows: $\forall K \in \mathcal{T}_{h} \forall i \in \mathcal{V}(K)$ set

$$
\begin{aligned}
u_{i, \max }^{\left(p_{1}, p_{2}\right)} & :=\max \left\{u_{K}^{\left(p_{1}, p_{2}\right)}, u_{i, \max }^{\left(p_{1}, p_{2}\right)}\right\}, \\
u_{i, \min }^{\left(p_{1}, p_{2}\right)} & :=\min \left\{u_{K}^{\left(p_{1}, p_{2}\right)}, u_{i, \min }^{\left(p_{1}, p_{2}\right)}\right\} .
\end{aligned}
$$

Remark 3. Hoteit et al. [19] generalize the above definition of $u_{i, \max }^{\left(p_{1}, p_{2}\right)}$ and $u_{i, \min }^{\left(p_{1}, p_{2}\right)}$ by introducing a free parameter that can be used to steer the amount of limiting. We do not use this trick to keep the method parameter-free.

Remark 4. The vertices located on the boundary of the domain require special treatment since the amount of information available at these vertices is limited. To prevent the bounds from becoming too restrictive, we set

$$
\begin{aligned}
& u_{i, \max }^{\left(p_{1}, p_{2}\right)}:=\max \left\{\hat{u}_{i}^{\left(p_{1}, p_{2}\right)}, u_{i, \max }^{\left(p_{1}, p_{2}\right)}\right\}, \\
& u_{i, \min }^{\left(p_{1}, p_{2}\right)}:=\min \left\{\hat{u}_{i}^{\left(p_{1}, p_{2}\right)}, u_{i, \min }^{\left(p_{1}, p_{2}\right)}\right\},
\end{aligned}
$$


where $\hat{u}_{i}^{\left(p_{1}, p_{2}\right)}$ is the upwind trace evaluated at the vertex $\left(x_{i}, y_{i}\right) \in \Gamma$. The value of $\hat{u}_{i}^{\left(p_{1}, p_{2}\right)}$ on $\Gamma_{\text {in }}$ is inferred from the Dirichlet boundary conditions.

If condition (32) holds for all vertices of $K$, no limiting is required for the gradient of $T_{K}^{\left(p_{1}, p_{2}\right)}$ and lower-order derivatives. If the inequality constraints are violated at any vertex, it is necessary to limit the gradient of $T_{K}^{\left(p_{1}, p_{2}\right)}$ and check the maximum principle for the Taylor polynomials of low-order derivatives. Thus, the limiting process must begin with the highest-order components and stop as soon as an unconstrained derivative satisfying (32) is found [21]. This hierarchical limiting strategy makes it possible to prevent spurious oscillations while preserving optimal accuracy at smooth peaks.

\section{Vertex-based limiter}

In the case of an explicit DG method, inequality constraints (32) can be readily enforced by limiting the Taylor degrees of freedom that define the fine-scale fluctuation. Given $u_{K}^{\left(p_{1}, p_{2}\right)}$, the objective is to find a correction factor $\alpha_{K}^{\left(p_{1}, p_{2}\right)} \in[0,1]$ such that the constrained Taylor polynomial

$$
\bar{T}_{K}^{\left(p_{1}, p_{2}\right)}(x, y)=u_{K}^{\left(p_{1}, p_{2}\right)}+\alpha_{K}^{\left(p_{1}, p_{2}\right)}\left\{u_{K}^{\left(p_{1}+1, p_{2}\right)} \frac{x-x_{c}}{\Delta x}+u_{K}^{\left(p_{1}, p_{2}+1\right)} \frac{y-y_{c}}{\Delta y}\right\}
$$

satisfies the inequality constraints

$$
u_{i, \min }^{\left(p_{1}, p_{2}\right)} \leq \bar{T}_{K}^{\left(p_{1}, p_{2}\right)}\left(x_{i}, y_{i}\right) \leq u_{i, \max }^{\left(p_{1}, p_{2}\right)}, \quad \forall i \in \mathcal{V}(K)
$$

Let $u_{i}^{\left(p_{1}, p_{2}\right)}=T_{K}^{\left(p_{1}, p_{2}\right)}\left(x_{i}, y_{i}\right)$ denote the unconstrained value of the Taylor polynomial at a vertex $\left(x_{i}, y_{i}\right)$. The correction factor $\alpha_{K}^{\left(p_{1}, p_{2}\right)}$ is defined by

$$
\alpha_{K}^{\left(p_{1}, p_{2}\right)}=\min _{i \in \mathcal{V}(K)}\left\{\begin{array}{cc}
\min \left\{1, \frac{u_{i, \max }^{\left(p_{1}, p_{2}\right)}-u_{K}^{\left(p_{1}, p_{2}\right)}}{\left.u_{i}^{\left(p_{1}, p_{2}\right)}-u_{K}^{\left(p_{1}, p_{2}\right)}\right\},},\right. & \text { if } u_{i}^{\left(p_{1}, p_{2}\right)}>u_{K}^{\left(p_{1}, p_{2}\right)} \\
1, & \text { if } u_{i}^{\left(p_{1}, p_{2}\right)}=u_{K}^{\left(p_{1}, p_{2}\right)} \\
\min \left\{1, \frac{u_{i, \min }^{\left(p_{1}, p_{2}\right)}-u_{K}^{\left(p_{1}, p_{2}\right)}}{u_{i}^{\left(p_{1}, p_{2}\right)}-u_{K}^{\left(p_{1}, p_{2}\right)}}\right\}, & \text { if } u_{i}^{\left(p_{1}, p_{2}\right)}<u_{K}^{\left(p_{1}, p_{2}\right)}
\end{array}\right.
$$

This limiting strategy was proposed by Barth and Jespersen [2] in the context of finite volume schemes using a different definition of $u_{i, \max }$ and $u_{i, \min }$. 
In the case of a $P_{2}$ approximation, the filtered polynomials are given by

\begin{tabular}{c|c}
$\left(p_{1}, p_{2}\right)$ & $\bar{T}^{\left(p_{1}, p_{2}\right)}(x, y) /\left(\Delta x^{p_{1}} \Delta y^{p_{2}}\right)$ \\
\hline$(0,0)$ & $\bar{u}_{K}+\alpha_{K}^{(0,0)}\left\{\left.\frac{\partial u}{\partial x}\right|_{c}\left(x-x_{c}\right)+\left.\frac{\partial u}{\partial y}\right|_{c}\left(y-y_{c}\right)\right\}$ \\
$(1,0)$ & $\left.\frac{\partial u}{\partial x}\right|_{c}+\alpha_{K}^{(1,0)}\left\{\left.\frac{\partial^{2} u}{\partial x^{2}}\right|_{c}\left(x-x_{c}\right)+\left.\frac{\partial^{2} u}{\partial x \partial y}\right|_{c}\left(y-y_{c}\right)\right\}$ \\
$(0,1)$ & $\left.\frac{\partial u}{\partial y}\right|_{c}+\alpha_{K}^{(0,1)}\left\{\left.\frac{\partial^{2} u}{\partial x \partial y}\right|_{c}\left(x-x_{c}\right)+\left.\frac{\partial^{2} u}{\partial y^{2}}\right|_{c}\left(y-y_{c}\right)\right\}$.
\end{tabular}

Since the mixed derivative appears in two rows of the above table, we multiply the second derivatives by the common correction factor

$$
\alpha_{K}^{(1)}:=\min \left\{\alpha_{K}^{(1,0)}, \alpha_{K}^{(0,1)}\right\} .
$$

In accordance with the assumption that derivatives of lower degree possess higher regularity, the correction factor for the gradient is given by

$$
\alpha_{K}^{(0)}:=\max \left\{\alpha_{K}^{(0,0)}, \alpha_{K}^{(1)}\right\} .
$$

In particular, this limiting strategy produces $\alpha_{K}^{(0)}=1$ at a smooth extremum, where the gradient varies smoothly and, therefore, $\alpha_{K}^{(1)}=1$. Numerical experiments confirm that the hierarchical limiting procedure does not degrade the rate of convergence even in the case of nonsmooth data.

The application of the vertex-based limiter to the derivatives of a twodimensional Taylor polynomial (20) yields the fine-scale fluctuation [24]

$$
\begin{aligned}
\bar{u}_{h}^{\prime}(x, y) & =\alpha_{K}^{(0)}\left\{\left.\frac{\partial u}{\partial x}\right|_{c}\left(x-x_{c}\right)+\left.\frac{\partial u}{\partial y}\right|_{c}\left(y-y_{c}\right)\right\} \\
& +\alpha_{K}^{(1)}\left\{\left.\frac{\partial^{2} u}{\partial x^{2}}\right|_{c}\left[\frac{\left(x-x_{c}\right)^{2}}{2}-\frac{\overline{\left(x-x_{c}\right)^{2}}}{2}\right]+\left.\frac{\partial^{2} u}{\partial y^{2}}\right|_{c}\left[\frac{\left(y-y_{c}\right)^{2}}{2}-\frac{\overline{\left(y-y_{c}\right)^{2}}}{2}\right]\right. \\
& \left.+\left.\frac{\partial^{2} u}{\partial x \partial y}\right|_{c}\left[\left(x-x_{c}\right)\left(y-y_{c}\right)-\overline{\left(x-x_{c}\right)\left(y-y_{c}\right)}\right]\right\},
\end{aligned}
$$

and the unconstrained quadratic shape function is overwritten by

$$
u_{h}(x, y):=\bar{u}_{h}+\bar{u}_{h}^{\prime}(x, y) .
$$

In general, we begin with the highest-order derivatives (cf. [21, 42]) and set

$$
\alpha_{K}^{(|p|)}:=\max _{|q|>|p|} \alpha_{K}^{(|q|)}, \quad|p| \geq 0
$$

As soon as $\alpha_{K}^{(|q|)}=1$ is encountered, no further limiting is performed since $\alpha_{K}^{(|p|)}=1$ for $|p| \leq|q|$. It is generally safe to use the correction factors given by (46) since solutions that require limiting cannot have smooth derivatives. 


\section{Constrained minimization}

A more general slope limiting strategy is based on solving a local minimization problem. Given the vector of Taylor degrees of freedom

$$
u_{K}^{\prime}=\left(u_{K}^{(1,0)}, u_{K}^{(0,1)}, u_{K}^{(2,0)}, u_{K}^{(0,2)}, u_{K}^{(1,1)}\right)^{T},
$$

the objective is to find $\bar{u}_{K}^{\prime}$ as close to $u_{K}^{\prime}$ as possible (in the sense to be defined below) without violating the imposed inequality constraints.

The optimization-based approach was employed, e.g., in the publications by Aizinger [1], Bochev et al. [6], Hoteit et al. [19], and Rider and Kothe [37]. As shown in $[1,6]$, conventional limiting is equivalent to solving a modified optimization problem with the same objective but simpler box constraints.

In this paper, we formulate the local inequality-constrained least squares problem for the vector of slope-limited derivatives $\bar{u}_{K}^{\prime}$ as follows:

$$
\left\{\begin{array}{l}
\min \left\|P\left(\bar{u}_{K}^{\prime}-u_{K}^{\prime}\right)\right\|_{2}^{2} \quad \text { subject to } \\
u_{i, \min } \leq T_{K}\left(x_{i}, y_{i}\right) \bar{u}_{K}^{\prime} \leq u_{i, \max }, \quad i \in \mathcal{V}(K)
\end{array}\right.
$$

where $\|\cdot\|_{2}$ is the Euclidean vector norm, $P$ is a matrix that defines the objective function for constrained minimization (see below) and $T\left(x_{i}, y_{i}\right) \bar{u}_{K}^{\prime}$ is the vector of components to be limited using the bounds

$$
u_{i, \max }=\left[\begin{array}{c}
u_{i, \max }^{(0,0)}-u_{K}^{(0,0)} \\
u_{i, \max }^{(1,0)}-u_{K}^{(1,0)} \\
u_{i, \max }^{(0,1)}-u_{K}^{(0,1)}
\end{array}\right], \quad u_{i, \min }=\left[\begin{array}{c}
u_{i, \min }^{(0,0)}-u_{K}^{(0,0)} \\
u_{i, \min }^{(1,0)}-u_{K}^{(1,0)} \\
u_{i, \min }^{(0,1)}-u_{K}^{(0,1)}
\end{array}\right] .
$$

In the case of $P_{2}$ elements, there are three constraints per vertex and

$$
T_{K}(x, y) u_{K}^{\prime}=\left[\begin{array}{c}
u_{K}^{(1,0)} \\
u_{K}^{(2,0)} \\
u_{K}^{(1,1)}
\end{array}\right] \frac{x-x_{c}}{\Delta x}+\left[\begin{array}{c}
u_{K}^{(0,1)} \\
u_{K}^{(1,1)} \\
u_{K}^{(0,2)}
\end{array}\right] \frac{y-y_{c}}{\Delta y} .
$$

If the objective function for (48) is defined using the identity matrix

$$
P=I
$$

then the difference between the values of $u_{K}^{\prime}$ and $\bar{u}_{K}^{\prime}$ is minimized. The sodefined objective is the same as that for the vertex-based slope limiter but 
the corresponding correction factors are determined individually for each derivative and are not required to lie between 0 and 1 .

Definition of the objective in terms of the reduced element mass matrix

$$
P=M_{K}^{\prime}
$$

minimizes the $L^{2}$-norm of the rejected fine-scale component $u_{h}^{\prime \prime}=u_{h}^{\prime}-\bar{u}_{h}^{\prime}$ subject to the imposed constraints. The optimization problem defined by

$$
P=A_{K}^{\prime}
$$

modifies the standard DG discretization of the convective term if it is found to produce undershoots or overshoots at the vertices of element $K$.

The local optimization problems are solved in a loop over elements. Given an unfiltered solution $u_{K}^{\prime}$, we check if the highest-order components of the vector $T_{K} u_{K}^{\prime}$ satisfy the imposed inequality constraints. If this is the case, we set $\bar{u}_{K}^{\prime}:=u_{K}^{\prime}$ and proceed to the next element. Otherwise, we solve the constrained least squares problem and overwrite $u_{K}^{\prime}$ by $\bar{u}_{K}^{\prime}$. In the current implementation, we use the NAG subroutine E04NCF as solver for (48).

The reasons for verification of constraints prior to solving the minimization problem are twofold. First, only a small number of "troubled cells" require limiting. In most elements, the solution to (48) is given by $\bar{u}_{K}^{\prime}=u_{K}^{\prime}$. Second, it is essential to avoid unnecessary limiting of low-order components in elements with smooth high-order components. As explained above, this is a prerequisite for preserving the order of accuracy at smooth extrema.

In our experience, the results are not very sensitive to the choice of the target matrix $P$, and there is no spectacular improvement in accuracy compared to the vertex-based limiter. However, constrained minimization makes it possible to avoid convergence problems associated with conventional slope limiting in implicit steady-state solvers (see Section 13). Moreover, the resid-

ual of the least squares problem may be used to quantify the amount of limiting and serve as an error indicator for adaptive mesh refinement.

\section{Explicit time stepping}

The semi-discrete DG scheme (15) can be discretized in time using an explicit or implicit method. In this section, we describe the implementation 
of slope limiting in explicit DG schemes. In particular, we consider the thirdorder strongly stability preserving (SSP) Runge-Kutta method [16]

$$
\begin{aligned}
& u_{K}^{(1)}=u_{K}^{n}+\Delta t M_{K}^{-1}\left[f_{K}^{n}-A_{K} u_{K}^{n}\right], \\
& u_{K}^{(2)}=\frac{3}{4} u_{K}^{n}+\frac{1}{4}\left(u_{K}^{(1)}+\Delta t M_{K}^{-1}\left[f_{K}^{(1)}-A_{K} u_{K}^{(1)}\right]\right), \\
& u_{K}^{n+1}=\frac{1}{3} u^{n}+\frac{2}{3}\left(u^{(2)}+\Delta t M_{K}^{-1}\left[f_{K}^{(2)}-A_{K} u_{K}^{(2)}\right]\right) .
\end{aligned}
$$

Like other SSP Runge-Kutta methods, this time-stepping scheme preserves the total variation diminishing (TVD) property of the underlying space discretization under certain time step restrictions. For a detailed presentation of such methods, we refer to the review paper by Gottlieb et al. [17].

The optimization-based approach is not to be recommended for explicit Runge-Kutta DG schemes since the vertex-based slope limiter presented in Section 7 produces essentially the same results at a lower cost [1]. In our implementation, the DG solution is constrained after each Runge-Kutta cycle. The solution values and the bounds are updated in a loop over elements. The correction factors $\alpha_{K}$ are calculated and the slope limiter is applied in a second loop over elements. Both loops are readily parallelizable.

In the case of a non-orthogonal Taylor basis, the non-vanishing off-diagonal entries of the consistent mass matrix $M_{K}$ give rise to an implicit coupling between the Taylor degrees of freedom in each element. The replacement of $M_{K}$ with the lumped mass matrix $M_{L}:=\operatorname{diag}\left\{m_{i i}\right\}$ preserves the mass of the DG solution but generates considerable errors, degrading the accuracy of high-order approximations. The contribution of $M_{K}-M_{L}$ does not cause any troubles in the case of smooth temporal variations but an implicit coupling between oscillatory time-dependent modes may give rise to spurious distortions of the solution profiles (see Fig. 2a,b below). To avoid the above side effects, we multiply the off-diagonal part of a non-diagonal mass matrix $M_{K}$ by the vector of pre-constrained time derivatives [25]. This filtering procedure can be interpreted as selective mass lumping.

Introducing the slope limiting operator $\Phi$, each step of the Runge-Kutta DG method for a general Taylor basis can be written as follows:

1. Given $u^{(k-1)}$, calculate the vector of discretized time derivatives

$$
\dot{u}_{K}^{(k)}=M_{K}^{-1}\left[f_{K}^{(k-1)}-A_{K} u_{K}^{(k-1)}\right] .
$$


2. Apply the vertex-based limiter $\Phi$ to the predictor $\dot{u}^{(k)}$ and calculate

$$
\tilde{u}_{K}^{(k)}=u_{K}^{(k-1)}+\Delta t M_{L}^{-1}\left[\left(M_{L}-M_{K}\right) \Phi \dot{u}_{K}^{(k)}+f_{K}^{(k-1)}-A_{K} u_{K}^{(k-1)}\right] .
$$

3. Apply the vertex-based limiter $\Phi$ to the convex average of $u^{n}$ and $\tilde{u}^{(k)}$

$$
u_{K}^{(k)}=\Phi\left(\omega_{k} u_{K}^{n}+\left(1-\omega_{k}\right) \tilde{u}_{K}^{(k)}\right),
$$

where $\omega_{k} \in[0,1]$ is the weight for step $k$ of the Runge-Kutta method.

The crucial step is the application of the slope limiting operator $\Phi$ to the vector of provisional time increments $\dot{u}_{K}^{(k)}$ at each RK step. If the Taylor basis is orthogonal $\left(M_{L}=M_{K}\right)$, then the contribution of $\Phi \dot{u}_{K}$ to (57) vanishes, so (56) should be omitted. If no limiting is performed in the second step $\left(\Phi \dot{u}_{K}=\dot{u}_{K}\right)$, the result is the consistent-mass DG approximation

$$
\tilde{u}_{K}^{(k)}=u_{K}^{(k-1)}+\Delta t M_{K}^{-1}\left[f_{K}^{(k-1)}-A_{K} u_{K}^{(k-1)}\right] .
$$

The application of the slope limiter to $\dot{u}_{K}^{(k)}$ eliminates non-smooth spatial variations in the discretized time derivatives, which improves the phase characteristics of the constrained DG scheme. In the case of a non-orthogonal Taylor basis, the extra cost is justified by a marked gain of accuracy.

The above approach to handling the off-diagonal part of the mass matrix can also be used to constrain transformations between an orthogonal (e.g., Dubiner) basis and a non-orthogonal Taylor basis. Such transformations are required in DG codes that use Taylor basis functions for limiting purposes only [36]. A non-diagonal transformation mass matrix may result in a harmful implicit coupling between the variations of Taylor degrees of freedom. Hence, the transformed increments may need to be constrained in the same fashion as the vector $\dot{u}_{K}$ in the above Runge-Kutta DG method.

\section{Implicit time stepping}

Slope limiting for implicit DG schemes is a far more challenging task than the explicit correction of derivatives in their explicit counterparts. In particular, the use of limiters may cause severe convergence problems in steady state computations [44]. Moreover, the number of degrees of freedom is much greater than in the case of continuous Galerkin methods, and interelement communication via jump terms results in wide stencils, which makes it difficult to design efficient iterative solvers for the global problem. 
A very promising approach to solving steady-state problems with implicit DG methods that employ Taylor basis functions is the $p$-multigrid method developed by Luo et al. [31, 33]. The underlying idea boils down to using a robust implicit solver for the lowest-order $\left(\bar{u}_{h}\right)$ component of the DG solution and an inexpensive explicit smoother on finer $p$-levels. The explicit nature of high-order corrections decouples the local problems for $u_{K}^{\prime}$ and makes it possible to perform limiting in the same way as in explicit DG schemes.

The idea of updating the coarse scales implicitly and fine scales explicitly is ideally suited for solving the nonlinear system associated with the implicit version of our variational multiscale DG method. Given the solution values from the previous iteration, we solve an implicit global problem for the coarsescale component $\bar{u}_{h}$, update the numerical fluxes and calculate the filtered fine-scale components by solving small local problems.

Without loss of generality we discretize the semi-discrete problem in (pseudo-)time using the backward Euler scheme. The fully discrete counterpart of the global coarse-scale problem (28) can be written as

$$
\bar{A} \bar{u}=\bar{b}\left(\bar{u}^{\prime}\right) .
$$

The discrete version of the fine-scale problem (29) is a system of the form

$$
A_{K}^{\prime} u_{K}^{\prime}=b_{K}^{\prime}\left(\bar{u}, \bar{u}^{\prime}\right)
$$

At each iteration or pseudo time-step, we update the DG solution as follows:

1. Given the vector of old limited derivatives $\bar{u}^{\prime}$, assemble $\bar{b}\left(\bar{u}^{\prime}\right)$ and update the mean values $\bar{u}$ by solving the coarse-scale problem (60).

2. In a loop over elements $K \in \mathcal{T}_{h}$, assemble $b_{K}^{\prime}\left(\bar{u}, \bar{u}^{\prime}\right)$ using the updated mean values $\bar{u}$ and old limited derivatives $\bar{u}^{\prime}$.

3. In a loop over elements $K \in \mathcal{T}_{h}$, solve the local problems (61) for the unconstrained derivatives $u_{K}^{\prime}$ and update the bounds.

4. In a loop over elements $K \in \mathcal{T}_{h}$, constrain the updated derivatives $u_{K}^{\prime}$ using the vertex-based limiter or constrained minimization.

We emphasize that the right-hand side of the local problem must be assembled using the filtered derivatives from the previous iteration. If steps 2 and 3 are performed in the same loop over elements, it is necessary to store $\bar{u}^{\prime}$ and $u^{\prime}$ as two separate vectors to avoid unintended overwriting. 
If steady-state solutions are of interest, the consistent mass matrix $M_{K}^{\prime}$ may be replaced with its diagonal part $M_{L}^{\prime}$. Interestingly enough, the nonorthogonality of the Taylor basis does not seem to have any detrimental effect on the accuracy of slope-limited solutions to unsteady transport equations. In contrast to the explicit Runge-Kutta DG scheme presented in the previous section, it is generally safe to use $M_{K}^{\prime}$ in the backward Euler DG scheme.

The global coarse problem has the structure of an upwind finite volume method. Hence, efficient iterative solvers are readily available. We solve system (60) using a matrix-free BiCGSTAB method with a diagonal pre-

conditioner. The residual assembly procedure involves element-by-element computations of matrix-vector products and numerical integration of the flux function in a loop over the edges/faces of each element $K \in \mathcal{T}_{h}$.

Remark 5. We also mention the possibility of numbering the elements in a way that produces a triangular matrix $\bar{A}$ and makes it possible to solve the coarse-scale problem (61) using forward or backward substitution.

In our experience, the above algorithm is very robust and does not impose any restrictions on the (pseudo)-time step. Moreover, it converges to steady state solutions if all components of $u_{K}^{\prime}$ are limited using the optimizationbased approach. The deactivation of limiting for lower-order components in elements with smooth high-order components produces a marked improvement in accuracy but prevents the residuals of the stationary coarse-scale problem from becoming smaller than a certain threshold, although the solutions "converge" in the eyeball norm and are free of visible oscillations.

\section{Reconstruction techniques}

Another implication of the multiscale nature of the DG method is the possibility of using postprocessing techniques as an inexpensive "subgrid scale model" for the fine-scale components. Reconstruction of derivatives has recently become a popular tool for reducing the number of degrees of freedom in DG methods $[12,13,34]$. In a DG scheme labeled $P_{n} P_{m}$, the shape function is defined using polynomials of degree $n$, while the fluxes are calculated using polynomials of degree $m$. The case $P_{0} P_{m}$ corresponds to a finite volume scheme, while $P_{m} P_{m}$ is the standard DG approximation. Numerical studies in Section 13 confirm that $P_{m-1} P_{m}$ may be as accurate as $P_{m} P_{m}$ for $m=1,2$.

The derivative reconstruction techniques proposed by Dumbser et al. [12, 13] and Luo et al. [34] are based on patch recovery. Given the DG solution 
restricted to a stencil of neighbor elements, the high-order reconstruction is obtained using an $L^{2}$ projection or least squares fitting. In our implementation of the $P_{m-1} P_{m}$ method, the piecewise-constant partial derivatives of degree $m-1$ are $L^{2}$-projected into the space $W_{h}$ of continuous $P_{1}$ or $Q_{1}$ finite

elements. Partial derivatives of degree $m$ are calculated by differentiating the resulting linear/multilinear shape function at the center of $K \in \mathcal{T}_{h}$.

The global $L^{2}$ projection $u_{h} \in W_{h}$ of a given function $u$ is defined by

$$
\int_{\Omega} w_{h} u_{h} \mathrm{~d} \mathbf{x}=\sum_{K \in \mathcal{T}_{h}} \int_{K} w_{h} u \mathrm{~d} \mathbf{x}, \quad \forall w_{h} \in W_{h} .
$$

The values of the continuous approximation $u_{h}$ at the vertices of the mesh are determined by solving a linear system of the form $M_{C} u=r$, where $M_{C}$ is the consistent mass matrix associated with the finite element space $W_{h}$.

Reconstructions of this kind can also be used to design parameter-free troubled cell markers and regularity indicators for $h p$-adaptivity. Derivatives of degree $|p| \geq 0$ may be regarded as smooth if the (discontinuous) higherorder reconstruction does not create new maxima or minima. In two recent publications [4, 28], we used this criterion to estimate the local smoothness and adjust the polynomial degree in continuous Galerkin methods. The deactivation of limiting in regions of high regularity was found to deliver optimal accuracy at smooth extrema without generating undershoots or overshoots elsewhere. For details, the interested reader is referred to $[4,28]$.

\section{Compressible Euler equations}

Of course, the above design principles are not restricted to DG schemes for the linear convection equation. In this section, we outline a generalization of the (explicit) algorithm [24] to the Euler equations of gas dynamics

$$
\begin{aligned}
\frac{\partial \rho}{\partial t}+\nabla \cdot(\rho \mathbf{v}) & =0 \\
\frac{\partial(\rho \mathbf{v})}{\partial t}+\nabla \cdot(\rho \mathbf{v} \otimes \mathbf{v}+p \mathcal{I}) & =0 \\
\frac{\partial(\rho E)}{\partial t}+\nabla \cdot(\rho E \mathbf{v}+p \mathbf{v}) & =0
\end{aligned}
$$

where $\rho$ is the density, $\mathbf{v}$ is the velocity, $p$ is the pressure, $E$ is the total energy, and $\mathcal{I}$ denotes the identity tensor. The equation of state for an ideal 
polytropic gas with the heat capacity ratio $\gamma$ reads

$$
p=(\gamma-1)\left(\rho E-\frac{\rho|\mathbf{v}|^{2}}{2}\right) .
$$

The Euler equations represent a nonlinear hyperbolic system of the form

$$
\frac{\partial U}{\partial t}+\nabla \cdot \mathbf{F}=0
$$

where

$$
U=\left[\begin{array}{c}
\rho \\
\rho \mathbf{v} \\
\rho E
\end{array}\right], \quad \mathbf{F}=\left[\begin{array}{c}
\rho \mathbf{v} \\
\rho \mathbf{v} \otimes \mathbf{v}+p \mathcal{I} \\
\rho E \mathbf{v}+p \mathbf{v}
\end{array}\right]
$$

The local problem associated with an element $K \in \mathcal{T}_{h}$ is given by

$$
\int_{K}\left(w \frac{\partial U}{\partial t}-\nabla w \cdot \mathbf{F}(U)\right) \mathrm{d} \mathbf{x}+\int_{\partial K} w \hat{\mathbf{F}}\left(U_{L}, U_{R}\right) \cdot \mathbf{n} \mathrm{d} s=0, \quad \forall w \in V .
$$

The flux $\hat{\mathbf{F}}\left(U_{L}, U_{R}\right)$ is defined using an approximate solution to the Riemann problem with the interior state $U_{L}=U^{+}$and exterior state $U_{R}=U^{-}$.

\subsection{Boundary conditions}

On a common edge/face of two mesh elements, the states $U_{L}$ and $U_{R}$ are defined as the one-sided traces of the (possibly discontinuous) solution. On the boundary $\Gamma$ of the computational domain $\Omega$, the components of $U_{R}$ are determined using $U_{L}$ and the characteristic boundary conditions. At a supersonic inlet, $U_{R}:=U_{\infty}$ is the vector of prescribed free stream values. At a supersonic outlet, we set $U_{R}:=U_{L}$. On a reflecting solid wall, we reverse the sign of the normal velocity by setting $(\rho \mathbf{v})_{R}:=(\rho \mathbf{v})_{L}-2 \mathbf{n}(\rho \mathbf{v})_{L} \cdot \mathbf{n}$. We refer to $[14,26,40]$ for details and other types of boundary conditions.

\subsection{Approximate Riemann solvers}

The choice of the numerical flux function $\hat{\mathbf{F}}$ must guarantee consistency

$$
\hat{\mathbf{F}}(U, U)=\mathbf{F}(U)
$$

and monotonicity of the coarse-scale (piecewise-constant) DG approximation.

A particularly simple formula is the Lax-Friedrichs / Rusanov flux

$$
\hat{\mathbf{F}}\left(U_{L}, U_{R}\right)=\frac{\mathbf{F}\left(U_{L}\right)+\mathbf{F}\left(U_{R}\right)}{2}-\max \left\{s_{L}, s_{R}\right\}\left(U_{R}-U_{L}\right),
$$


where $s_{L}$ and $s_{R}$ are defined as the fastest characteristic speeds. In the case of the compressible Euler equations, the solution to the local Riemann problem is a superposition of waves moving at speeds $\mathbf{n} \cdot \mathbf{v}$ and $\mathbf{n} \cdot \mathbf{v} \pm c$, where $c=\sqrt{\gamma p / \rho}$ is the local speed of sound. Hence, we have

$$
s_{L}=\left|\mathbf{n} \cdot \mathbf{v}_{L}\right|+c_{L}, \quad s_{R}=\left|\mathbf{n} \cdot \mathbf{v}_{R}\right|+c_{R} .
$$

The Lax-Friedrichs flux (69) is very robust but tends to produce excessive numerical dissipation. As an alternative, we consider the HLL flux [18, 39]

$$
\hat{\mathbf{F}}\left(U_{L}, U_{R}\right)=\left\{\begin{array}{cl}
\mathbf{F}\left(U_{L}\right), & \text { if } s_{L}>0, \\
\mathbf{F}\left(U_{R}\right), & \text { if } s_{R}<0, \\
\frac{s_{R} \mathbf{F}_{L}}{s_{R}-s_{L}}-\frac{s_{L} \mathbf{F}_{R}}{s_{R}-s_{L}}+\frac{s_{L} s_{R}}{s_{R}-s_{L}}\left(U_{R}-U_{L}\right), & \text { otherwise }
\end{array}\right.
$$

where

$$
\begin{aligned}
s_{L} & =\min \left\{\mathbf{n} \cdot \mathbf{v}_{L}, \mathbf{n} \cdot \mathbf{v}_{R}\right\}-\max \left\{c_{L}, c_{R}\right\} \\
s_{R} & =\max \left\{\mathbf{n} \cdot \mathbf{v}_{L}, \mathbf{n} \cdot \mathbf{v}_{R}\right\}+\max \left\{c_{L}, c_{R}\right\} .
\end{aligned}
$$

For a review of popular approximate Riemann solvers, we refer to Toro [39].

\subsection{Compatible gradient limiting}

Slope limiting for systems of conservation laws requires special care since certain derived quantities (e.g., v, $E, p$ ) may exhibit undershoots/overshoots even if the conserved quantities (i.e., $\rho, \rho \mathbf{v}, \rho E$ ) are bounded by the means. A limiting procedure that preserves monotonicity of nonconservative quantities of interest is called compatible [43]. The design of compatible limiting techniques involves a transformation of variables. For example, Vilar [45] constrained the local characteristic variables using a generalization of the vertex-based slope limiter. Characteristic-based limiters for the onedimensional Euler equations are known to be compatible and very robust [26]. In the multidimensional case, waves may travel in infinitely many directions, whence the definition of characteristic variables is ambiguous. We remark that the inequality constraints for a vertex $\mathbf{x}_{i}$ must be defined using the same characteristic direction $\mathbf{n}_{i}$ for all transformed quantities.

In this paper, we propose another approach to enforcing compatibility in the gradient limiting procedure for the Euler equations. Applying the vertex-based limiter to a single component $\rho u$ of the solution vector $U$, we obtain the limited gradient $\alpha_{K}^{\rho u} \nabla(\rho u)_{K}\left(\mathbf{x}_{c}\right)$. By the product rule, we have

$$
\nabla u_{K}\left(\mathbf{x}_{c}\right)=\frac{\alpha_{K}^{\rho u} \nabla(\rho u)_{K}\left(\mathbf{x}_{c}\right)-u_{K}\left(\mathbf{x}_{c}\right) \alpha_{K}^{\rho} \nabla \rho_{K}\left(\mathbf{x}_{c}\right)}{\rho_{K}\left(\mathbf{x}_{c}\right)} .
$$


Next, we calculate the bounds for the values of $u$ at the vertices of the mesh and apply the vertex-based limiter to the gradient $\nabla u_{K}\left(\mathbf{x}_{c}\right)$ defined in (71). Finally, the gradient of the conserved quantity $\rho u$ is corrected as follows:

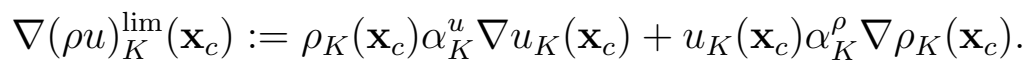

In the below numerical study, we use this gradient limiting procedure to constrain each velocity component and the energy density. In our experience, the proposed amendment leads to a marked improvement in situations when segregated limiting produces spurious ripples. Moreover, the cost of limiting $\mathbf{v}$ and $E$ is not as high as that of a characteristic-based slope limiter.

\section{Numerical studies}

In this section, we perform a numerical study of the presented schemes for the linear convection equation and for the Euler equations. For visualization purposes, all solutions are projected into the space of continuous linears or bilinears using the $L^{2}$ projection described in Section 11. Mass lumping is employed to prevent the projection operator from producing undershoots or overshoots. The use of limiting in $L^{2}$ projections is discussed in $[4,27]$.

\subsection{Solid body rotation}

The solid body rotation benchmark proposed by LeVeque [30] is often used to evaluate numerical advection schemes. In this test, the velocity field $\mathbf{v}$ for the two-dimensional convection equation (1) is given by

$$
\mathbf{v}(x, y)=(0.5-y, x-0.5)
$$

This formula describes a counterclockwise rotation about the center of the domain $\Omega=(0,1) \times(0,1)$. After each full revolution, the exact solution $u$ coincides with the initial data $u_{0}$. The objective of the numerical study is to assess the ability of the given scheme to preserve the shape of $u_{0}$.

Following LeVeque [30], we consider the initial configuration displayed in Fig. 1a. The geometry of each body is described by a function $G(x, y)$ defined on a circle of radius $r_{0}=0.15$ centered at some point $\left(x_{0}, y_{0}\right)$. Let

$$
r(x, y)=\frac{1}{r_{0}} \sqrt{\left(x-x_{0}\right)^{2}+\left(y-y_{0}\right)^{2}}
$$

be the normalized distance from $\left(x_{0}, y_{0}\right)$. Then $r(x, y) \leq 1$ inside the circle. 
The slotted cylinder is centered at the point $\left(x_{0}, y_{0}\right)=(0.5,0.75)$ and

$$
G(x, y)= \begin{cases}1 & \text { if }\left|x-x_{0}\right| \geq 0.025 \text { or } y \geq 0.85 \\ 0 & \text { otherwise }\end{cases}
$$

The cone is centered at $\left(x_{0}, y_{0}\right)=(0.5,0.25)$, and its shape is given by

$$
G(x, y)=1-r(x, y) .
$$

The hump is centered at $\left(x_{0}, y_{0}\right)=(0.25,0.5)$, and the shape function is

$$
G(x, y)=\frac{1+\cos (\pi r(x, y))}{4} .
$$

The above test problem is solved using explicit DG approximations with orthogonal and non-orthogonal Taylor bases. Of course, not only cell averages but also the derivatives must be initialized. The numerical solutions presented in Fig. 1 were calculated using the RKDG method and the vertexbased limiter on a uniform mesh of rectangular elements. The mesh size and time step for this simulation are given by $h=1 / 128$ and $\Delta t=10^{-3}$, respectively. The $P_{0}$ approximation produces the diffusive solution shown in Fig. 1b. The limited $P_{1}$ approximation is more accurate but exhibits peak clipping (Fig. 1c), whereas the $P_{2}$ version (Fig. 1d) preserves the two peaks remarkably well. The global errors $E_{2}=\left\|u-u_{h}\right\|_{2}$ are measured in the $L^{2}$ norm at the final time $t=2 \pi$. They are calculated using the original DG solution rather than the continuous projections presented in Fig. 1.

The results for the slope-limited $P_{1}$ and $P_{2}$ approximations on a triangular mesh with the same vertices are displayed in Fig. 2. In this case, the Taylor basis (21) is non-orthogonal, which means that there are implicit links between the derivatives of the DG solution in each element. The computation of $\tilde{u}_{K}^{(k)}$ using (58) without limiting produces the inaccurate solutions shown in Fig. 2a-b. Replacing the consistent mass matrix $M_{K}$ with its diagonal part $M_{L}$, one obtains the results in Fig. 2c-d. Note that the $P_{2}$ solution is just marginally better than its $P_{1}$ counterpart and also exhibits peak clipping. The results shown in Fig. 2e-f were calculated with algorithm (56)-(57). The application of the vertex-based limiter to the vector of time derivatives makes it possible to recover the high accuracy of the $P_{2}$ approximation in smooth regions, and the results are even better than those in Fig. 1c-d.

For a better visual comparison of the solution profiles, we present 4 cutlines of the initial and final solutions in Figs 3-5. The difference between the 
(a) Initial/exact solution, $E_{2}=0.0$

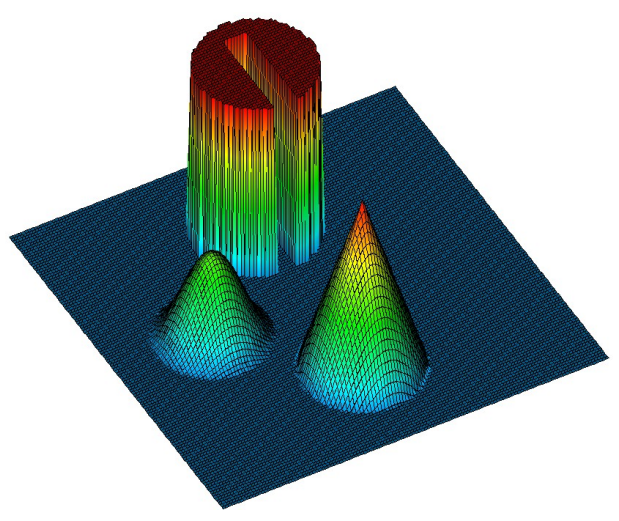

(c) $P_{1}$ elements, $E_{2}=7.19 \mathrm{e}-2$

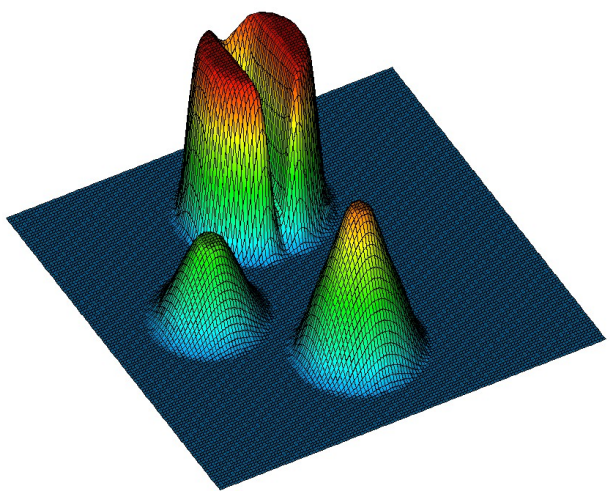

(b) $P_{0}$ elements, $E_{2}=1.80 \mathrm{e}-1$

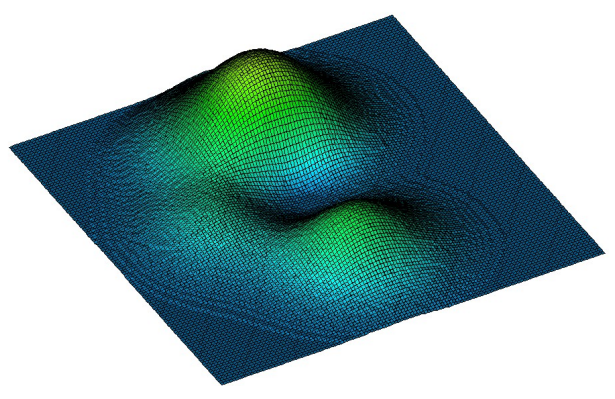

(d) $P_{2}$ elements, $E_{2}=6.60 \mathrm{e}-2$

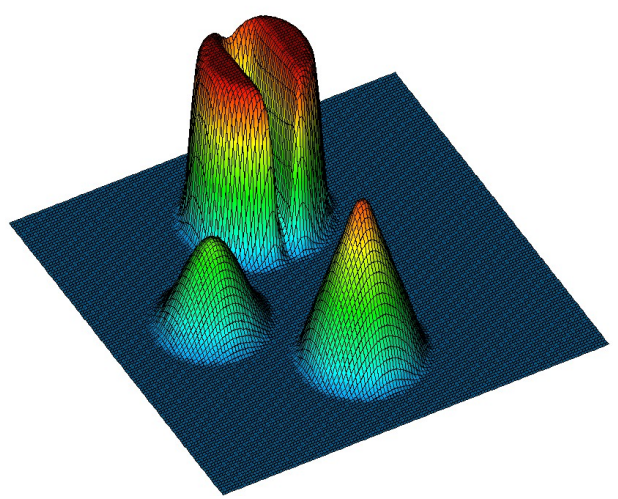

Figure 1: Solid body rotation, simulation on a rectangular mesh, $t=2 \pi$.

$P_{1}$ and $P_{2}$ approximations is particularly pronounced near the two peaks. Note that the $P_{2}$ version resolves the smooth hump $(x=0.25)$ perfectly if the Taylor basis is orthogonal (Fig. 3c) or if the off-diagonal part of the mass matrix is applied to the vector of limited time derivatives (Fig. 5c). The peak of the cone $(y=0.25)$ is also preserved much better than in the $P_{1}$ version. In the neighborhood of the slotted cylinder $(y=0.75)$, the vertex-based limiter switches to the monotone $P_{0}$ approximation. For this reason, the differences between the limited $P_{1}$ and $P_{2}$ solutions are marginal in this region. 
(a) $P_{1} /$ consistent mass, $E_{2}=1.33 \mathrm{e}-1$

(b) $P_{2} /$ consistent mass, $E_{2}=1.11 \mathrm{e}-1$

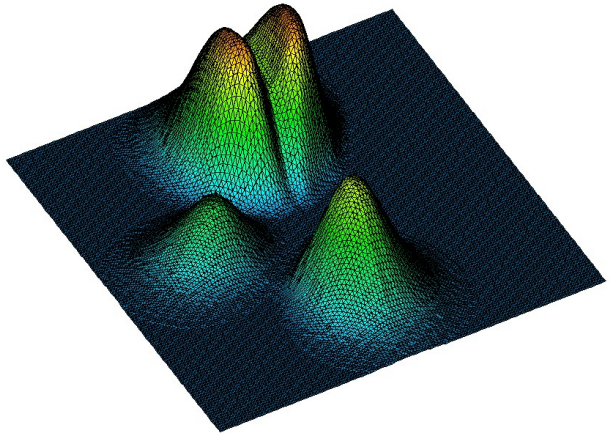

(c) $P_{1} /$ lumped mass, $E_{2}=6.81 \mathrm{e}-2$

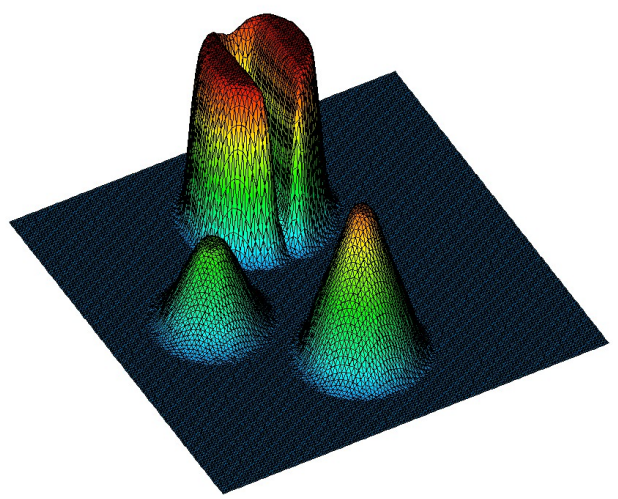

(e) $P_{1} /$ limited mass, $E_{2}=6.50 \mathrm{e}-2$

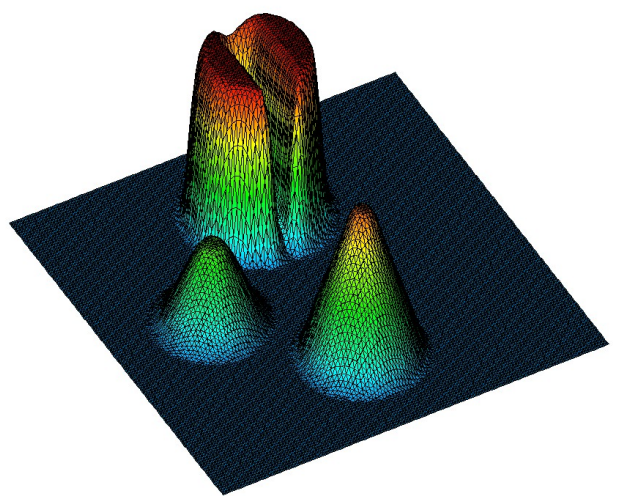

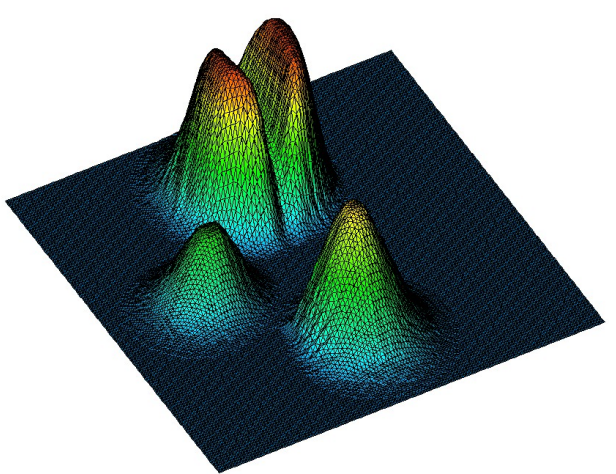

(d) $P_{2} /$ lumped mass, $E_{2}=6.70 \mathrm{e}-2$

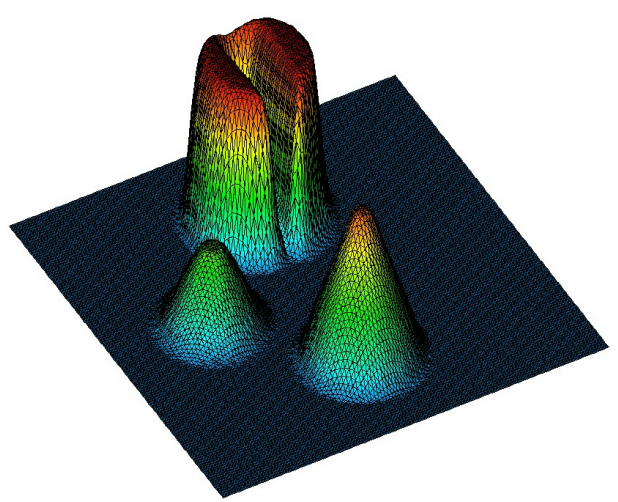

(f) $P_{2} /$ limited mass, $E_{2}=6.05 \mathrm{e}-2$

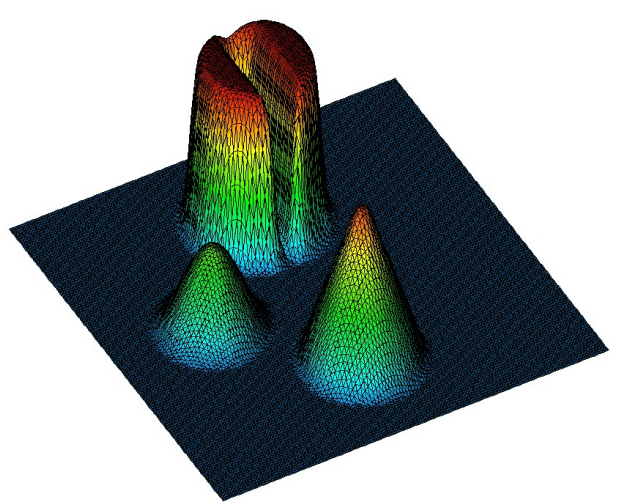

Figure 2: Solid body rotation, simulation on a triangular mesh, $t=2 \pi$. 
(a) $y=0.25$

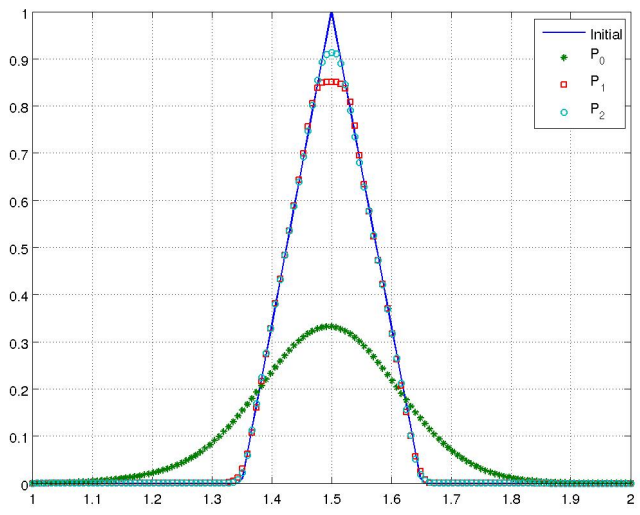

(c) $x=0.25$

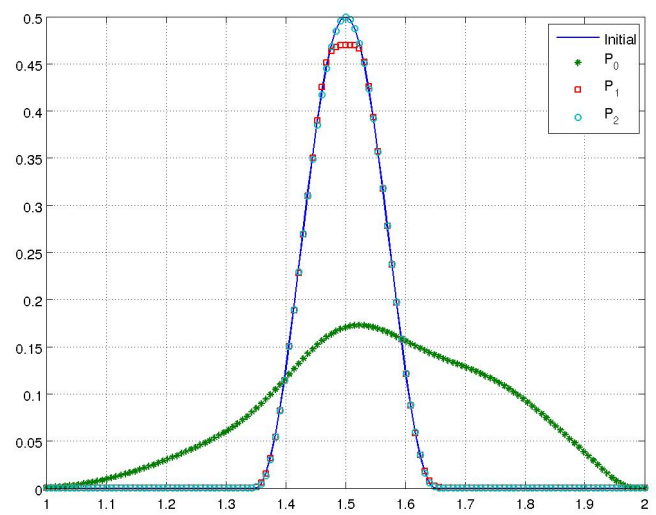

(b) $y=0.75$

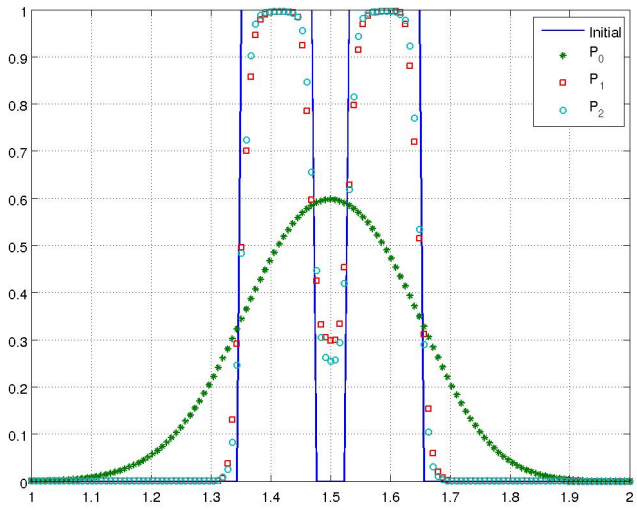

(d) $x=0.5$

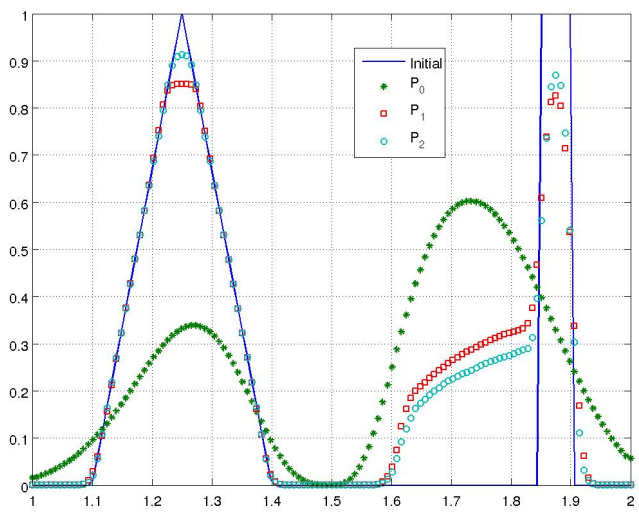

Figure 3: Cutlines of the DG solutions on the triangular mesh, $t=2 \pi$.

The results produced by the $P_{m-1} P_{m}$ algorithm that approximates the DG solution using polynomials of degree $m-1$ and reconstructs derivatives of degree $m$ to calculate the fluxes are displayed in Fig. 6. The $L^{2}$ errors $\left(E_{2}=7.99 \mathrm{e}-2\right.$ for $P_{0} P_{1}$ and $E_{2}=6.16 \mathrm{e}-2$ for $\left.P_{1} P_{2}\right)$ are just marginally larger than those produced by the $P_{m} P_{m}$ version for $m=1,2$. This confirms that reconstruction techniques constitute a viable alternative to computation of higher-order derivatives. However, this approach does not offer significant savings in explicit DG methods since the cost of solving the local fine-scale problems for the $P_{m} P_{m}$ discretization is just as low or even lower. 
(a) $y=0.25$

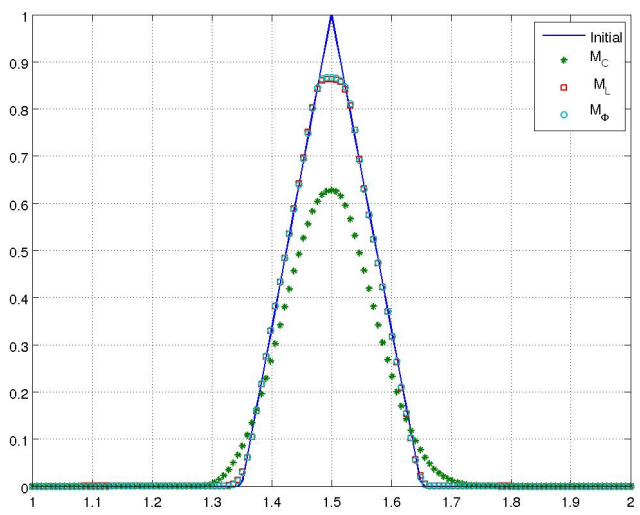

(c) $x=0.25$

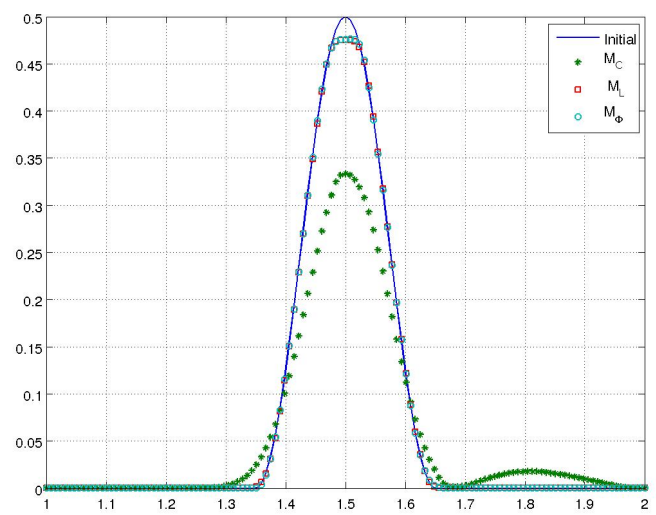

(b) $y=0.75$

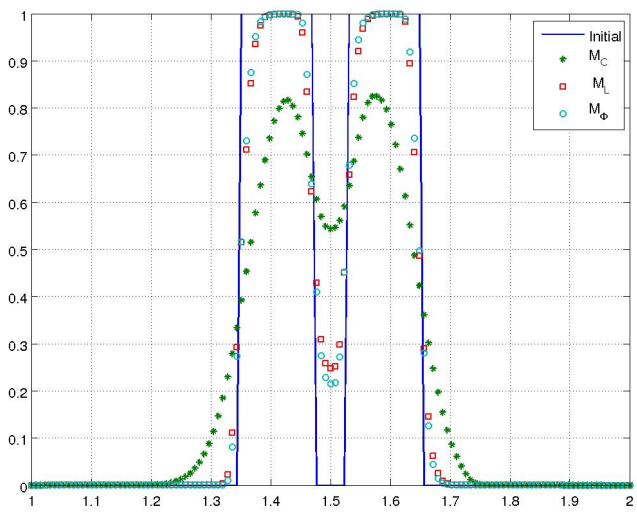

(d) $x=0.5$

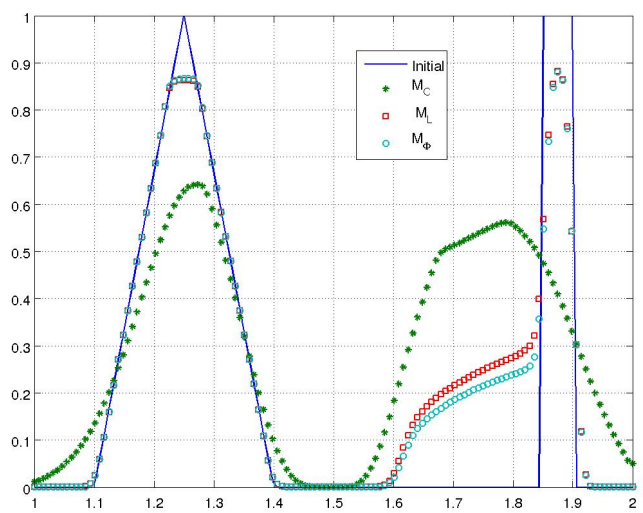

Figure 4: Cutlines of the $P_{1}$ solutions on the triangular mesh, $t=2 \pi$. Results obtained with the consistent mass $\left(M_{C}\right)$, lumped mass $\left(M_{L}\right)$, and limited mass $\left(M_{\Phi}\right)$.

\subsection{Circular convection}

In the second test, the steady-state counterpart of equation (1) is solved in the rectangular domain $\Omega=(0,2) \times(0,1)$. The exact solution and inflow boundary conditions for $\mathbf{v}(x, y)=(y, 1-x)$ are given by the formula

$$
u(x, y)= \begin{cases}1, & \text { if } 0.2 \leq r \leq 0.4 \\ \frac{1}{4}\left[1+\cos \left(\pi \frac{r-0.65}{0.15}\right)\right], & \text { if } 0.5 \leq r \leq 0.8 \\ 0, & \text { otherwise }\end{cases}
$$


(a) $y=0.25$

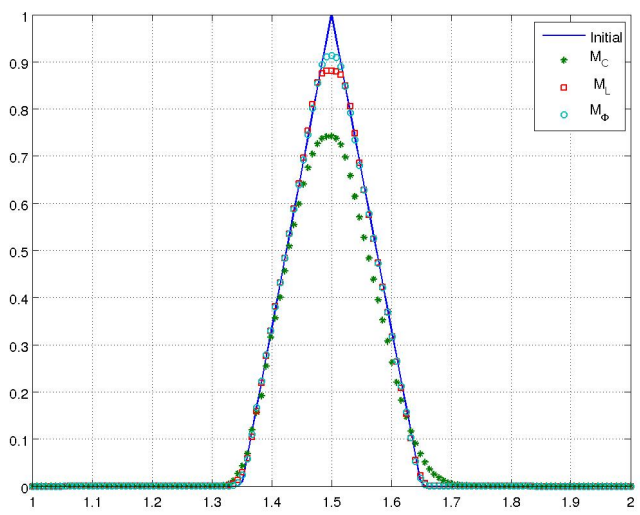

(c) $x=0.25$

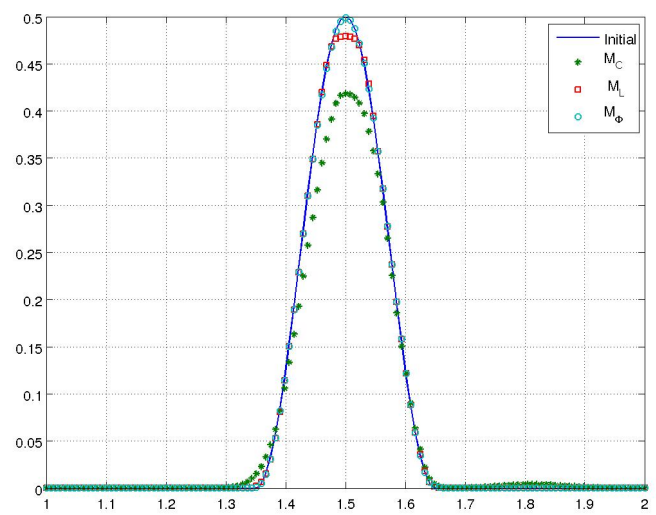

(b) $y=0.75$

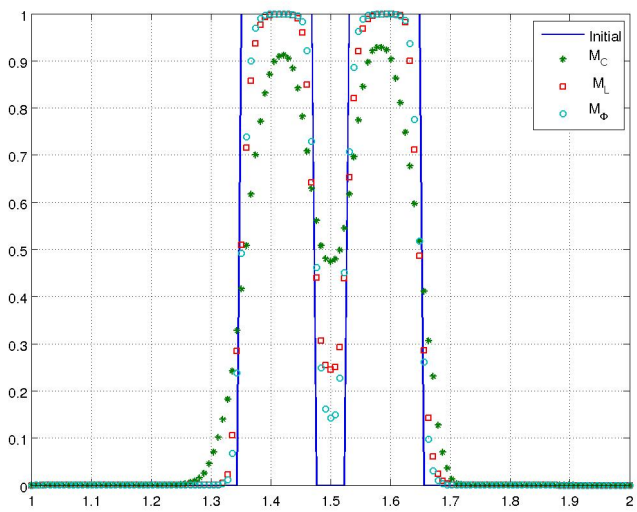

(d) $x=0.5$

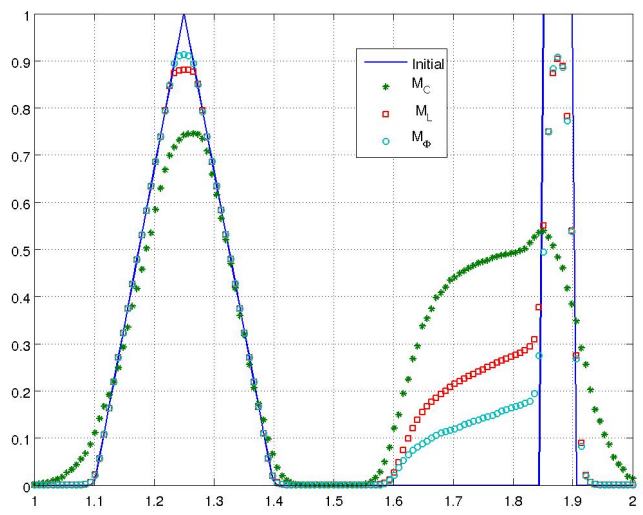

Figure 5: Cutlines of the $P_{2}$ solutions on the triangular mesh, $t=2 \pi$. Results obtained with the consistent mass $\left(M_{C}\right)$, lumped mass $\left(M_{L}\right)$, and limited mass $\left(M_{\Phi}\right)$.

where $r=\sqrt{(x-1)^{2}+y^{2}}$ is the distance from the reference point $(1.0,0.0)$.

Steady-state computations are performed with the $P_{1}$ and $P_{2}$ versions of the backward Euler DG scheme. The nonlinear system is solved using the two-level iterative solver presented in Section 10. The mesh is rectangular with $h=1 / 128$. The pseudo-time step for this simulation is as large as $\Delta t=1.0$. The stationary solutions displayed in Fig. 7 were obtained using constrained optimization (CO) with the target matrix $P=A_{K}^{\prime}$. The vertexbased (VB) limiter produces similar results (not shown here). The results of 
(a) $P_{0} P_{1}$ scheme, $E_{2}=7.99 \mathrm{e}-2$

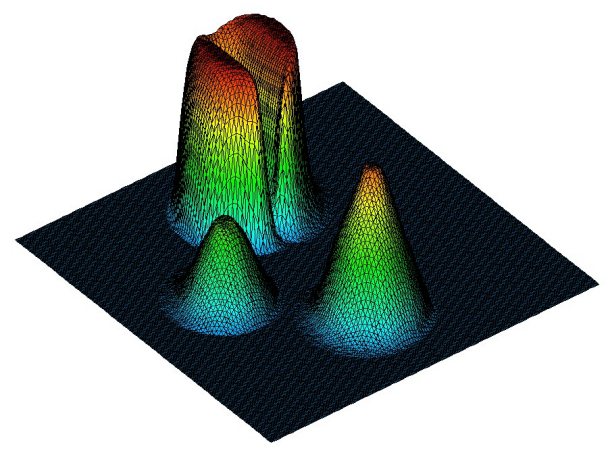

(b) $P_{1} P_{2}$ scheme, $E_{2}=6.16 \mathrm{e}-2$

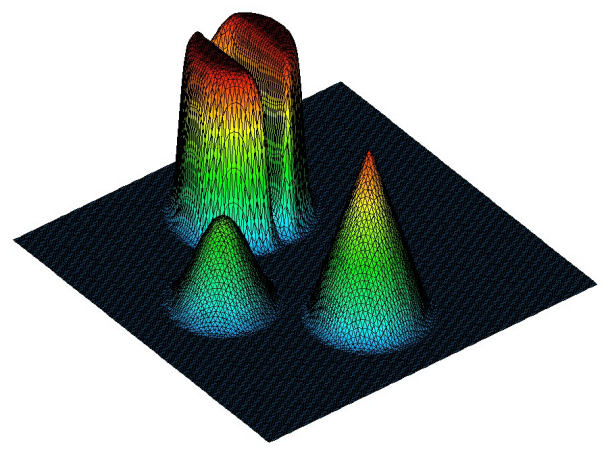

Figure 6: Solid body rotation, $P_{m-1} P_{m}$ methods on a triangular mesh, $t=2 \pi$.

(a) $P_{1}$-VB, $E_{2}=6.28 \mathrm{e}-2$

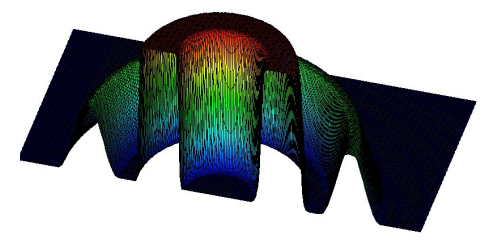

(b) $P_{2}$-VB, $E_{2}=5.88 \mathrm{e}-2$

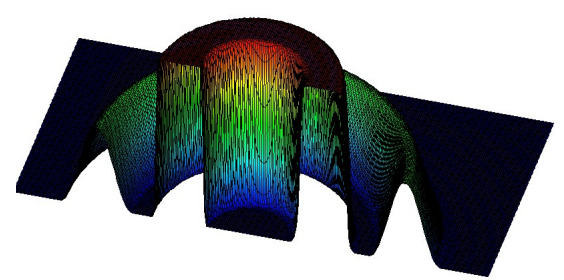

Figure 7: Circular convection, constrained minimization on a rectangular mesh.

a grid convergence study for both methods are summarized in Table 1 .

The $P_{2}$ approximation produces smaller errors than the $P_{1}$ scheme, while the way in which the bounds are enforced has hardly any influence on the results. At the same time, large differences are observed in the evolution of the steady state residuals of the coarse-scale problem. As shown by the $P_{1}$ convergence history presented in Fig. 8, the $\mathrm{CO}$ algorithm drives the Euclidean norm of the residual towards machine zero, while conventional VB slope limiting prevents it from decreasing any further after reaching a certain threshold. In the $P_{2}$ version of the implicit DG scheme, the residuals behave 


\begin{tabular}{|c|c|c|c|c|}
\hline & \multicolumn{2}{|c|}{ DG-VB } & \multicolumn{2}{c|}{ DG-CO } \\
\hline$h$ & $P_{1}$ & $P_{2}$ & $P_{1}$ & $P_{2}$ \\
\hline $1 / 16$ & $2.08 \mathrm{e}-01$ & $1.67 \mathrm{e}-01$ & $2.06 \mathrm{e}-01$ & $1.67 \mathrm{e}-01$ \\
$1 / 32$ & $1.25 \mathrm{e}-01$ & $1.03 \mathrm{e}-01$ & $1.11 \mathrm{e}-01$ & $1.03 \mathrm{e}-01$ \\
$1 / 64$ & $9.75 \mathrm{e}-02$ & $7.65 \mathrm{e}-02$ & $8.16 \mathrm{e}-02$ & $7.65 \mathrm{e}-02$ \\
$1 / 128$ & $8.14 \mathrm{e}-02$ & $5.81 \mathrm{e}-02$ & $6.28 \mathrm{e}-02$ & $5.81 \mathrm{e}-02$ \\
\hline
\end{tabular}

Table 1: Circular convection: $E_{2}$ errors on four successively refined meshes.

in the same way as long as both first and second derivatives are constrained. The deactivation of $\mathrm{CO}$ in elements in which the inequality constraints hold for the second derivatives results in stagnation of steady state residuals but prevents degradation of accuracy at smooth extrema. Since VB deactivates itself in the same cells, the constrained $P_{2}$ solutions are virtually identical.

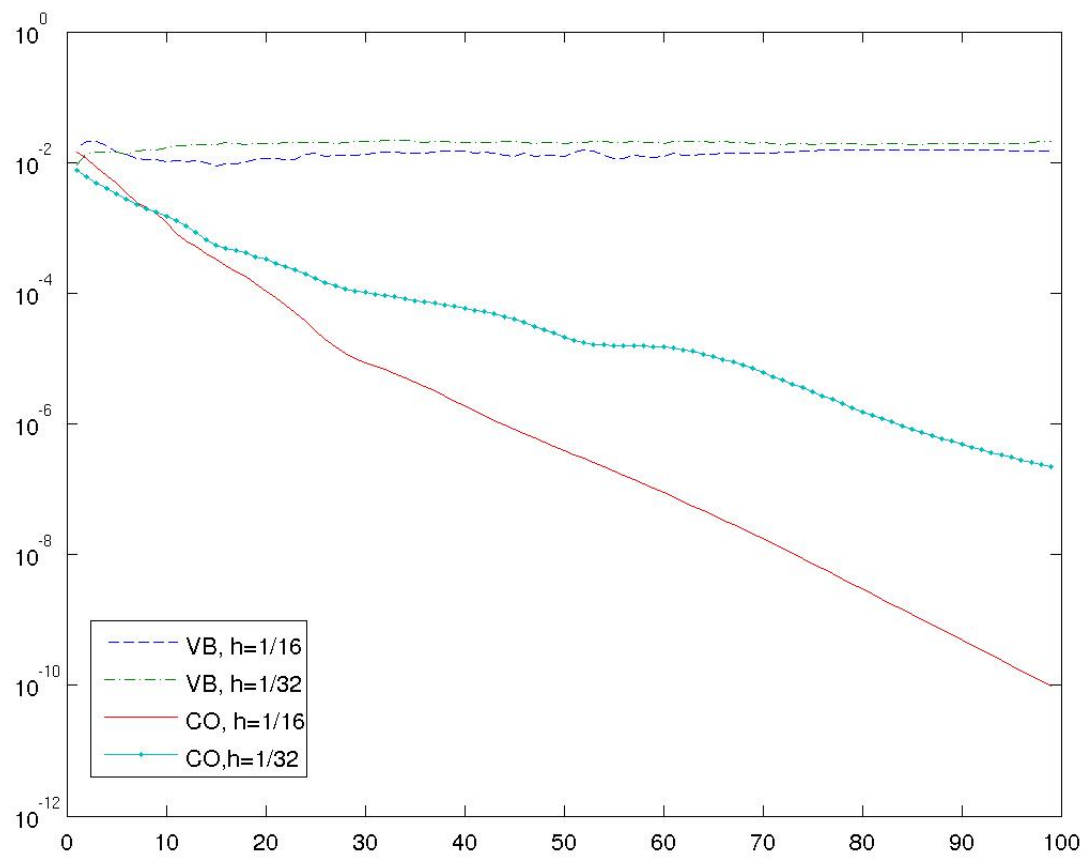

Figure 8: Circular convection, $P_{1}$ approximation, Euclidean norm of the steady-state residual (coarse-scale problem) vs. number of pseudo-time steps. 


\subsection{Shock tube problem}

Sod's shock tube problem [38] is a standard benchmark for the Euler equations of gas dynamics. The domain $\Omega=(0,1) \times(0,1)$ has reflective walls and is initially separated by a membrane into two sections. When the membrane is removed, the gas begins to flow into the region of lower pressure. The initial condition for the nonlinear Riemann problem is given by

$$
\left[\begin{array}{l}
\rho_{L} \\
\mathbf{v}_{L} \\
p_{L}
\end{array}\right]=\left[\begin{array}{l}
1.0 \\
0.0 \\
1.0
\end{array}\right], \quad\left[\begin{array}{l}
\rho_{R} \\
\mathbf{v}_{R} \\
p_{R}
\end{array}\right]=\left[\begin{array}{c}
0.125 \\
0.0 \\
0.1
\end{array}\right],
$$

where the subscripts refer to $\Omega_{L}=(0,0.5) \times(0,1)$ and $\Omega_{R}=(0.5,1) \times(0,1)$.

The removal of the membrane at $t=0$ releases a shock wave that propagates to the right with velocity satisfying the Rankine-Hugoniot conditions. All of the primitive variables are discontinuous across the shock which is followed by a contact discontinuity. The latter represents a moving interface between the regions of different densities but constant velocity and pressure. Furthermore, a rarefaction wave propagates in the opposite direction providing a smooth transition to the original values of the state variables in the left part of the domain. Hence, the 1D flow pattern in the shock tube is characterized by three waves traveling at different speeds.

The dashed lines in Fig. 9 depict the exact solution at the final time $t=0.231$. The solid lines show the numerical solutions calculated using the explicit DG method with $h=1 / 128$ and $\Delta t=10^{-3}$. The presented values of the $E_{2}$ error for the density field confirm that the Lax-Friedrichs flux (LF) produces more numerical dissipation than the HLL flux, especially in the case of a $P_{0}$ discretization (see Figs $9 \mathrm{a}, \mathrm{b}$ ). The difference between the slope-limited $P_{1}$ solutions is less pronounced. The results in Figs $9 \mathrm{c}, \mathrm{d}$ were calculated using the vertex-based limiter to constrain the conservative variables $\rho, \rho \mathbf{v}$, and $\rho E$ in a segregated manner. The unconstrained velocity and pressure exhibit undershoots and overshoots which carry over to the conservative variables. The activation of compatible gradient limiting (VBC) for the velocity and energy density (Section 12.3) leads to a further gain of accuracy and produces monotone solution profiles, as shown in Figs 9e,f.

\subsection{Radially symmetric Riemann problem}

The next benchmark is a radially symmetric $2 \mathrm{D}$ counterpart of the shock tube problem [29]. Before an impulsive start, an imaginary membrane sepa- 
(a) LF- $P_{0}, E_{2}^{\rho}=1.17 \mathrm{e}-3$

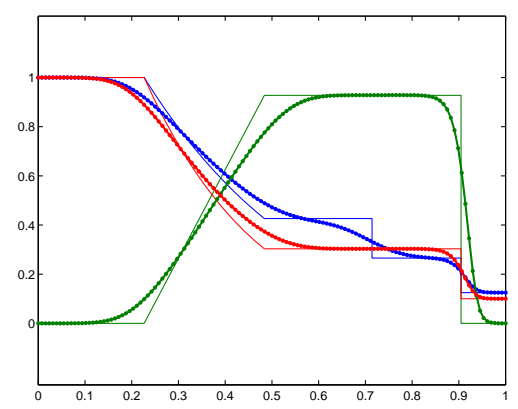

(c) $\mathrm{LF}-P_{1}-\mathrm{VB}, E_{2}^{\rho}=1.76 \mathrm{e}-4$

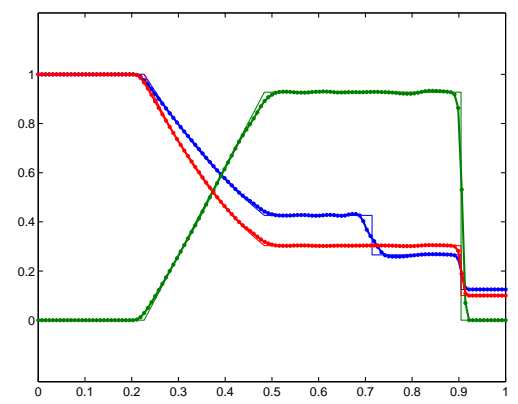

(e) LF- $P_{1}$-VBC, $E_{2}^{\rho}=1.41 \mathrm{e}-4$

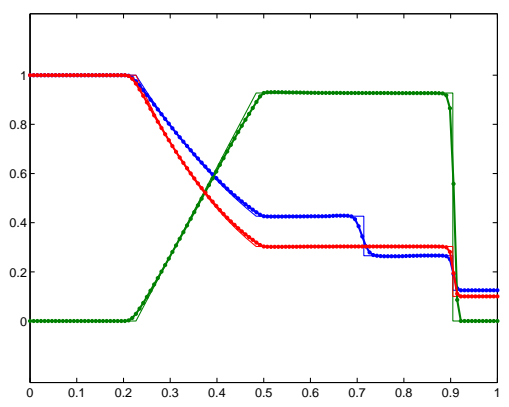

(b) HLL- $P_{0}, E_{2}^{\rho}=7.96 \mathrm{e}-4$

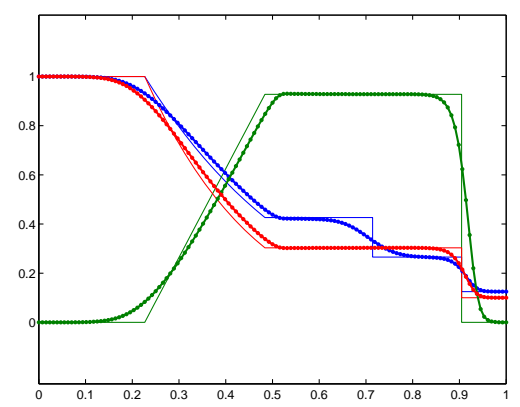

(d) HLL- $P_{1}$-VB, $E_{2}^{\rho}=1.56 \mathrm{e}-4$

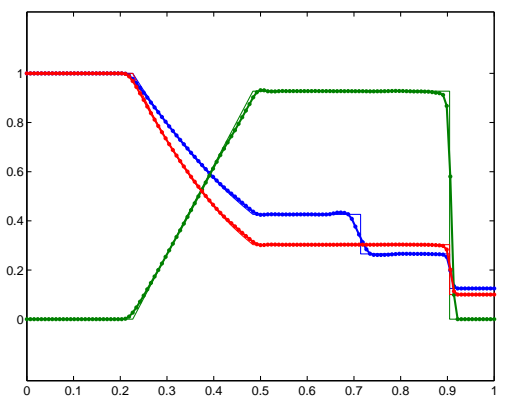

(f) HLL- $P_{1}$-VBC, $E_{2}^{\rho}=1.18 \mathrm{e}-4$

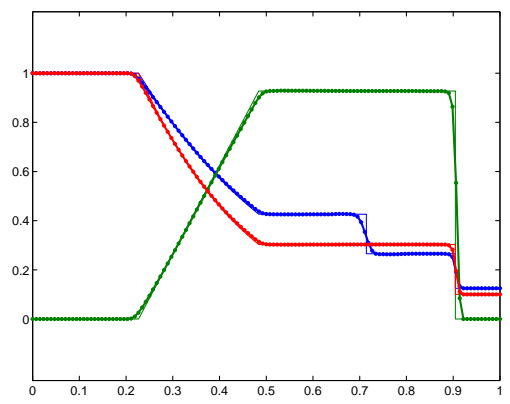

Figure 9: Shock tube problem: $h=1 / 128, \Delta t=10^{-3}$. Snapshots of the density (blue), velocity (green), and pressure (red) distribution along the line $y=0.5$ at $t=0.231$. 
rates the square domain $\Omega=(-0.5,0.5) \times(-0.5,0.5)$ into the subdomains

$$
\Omega_{L}=\left\{(x, y) \in \Omega \mid \sqrt{x^{2}+y^{2}}<0.13\right\}
$$

and $\Omega_{R}=\Omega \backslash \Omega_{L}$. Reflective boundary conditions are prescribed on $\Gamma$. The gas is initially at rest. Higher pressure and density are maintained inside $\Omega_{L}$ than outside. The interior and exterior states are given by

$$
\left[\begin{array}{c}
\rho_{L} \\
\mathbf{v}_{L} \\
p_{L}
\end{array}\right]=\left[\begin{array}{c}
2.0 \\
0.0 \\
15.0
\end{array}\right], \quad\left[\begin{array}{c}
\rho_{R} \\
\mathbf{v}_{R} \\
p_{R}
\end{array}\right]=\left[\begin{array}{c}
1.0 \\
0.0 \\
1.0
\end{array}\right] .
$$

The abrupt removal of the membrane at $t=0$ gives rise to a radially expanding shock wave driven by the pressure difference. The challenge of this test is to capture the moving discontinuities while preserving radial symmetry.

Fig. 10 displays the isolines of the density distribution calculated with the $P_{0}$ and $P_{1}$ versions of the HLL-DG method using the same mesh and time step as in the previous example. The $P_{1}$ solution is constrained using the compatible gradient limiting strategy. The cutlines displayed in Fig. 11 show that the density profiles are perfectly symmetric and free of ripples.

\subsection{Double Mach reflection}

A more challenging test for the unsteady Euler equations is the double Mach reflection problem of Woodward and Colella [46] . In this benchmark, a Mach 10 shock impinges on a reflecting wall at the angle of $60^{\circ}$ degrees. The computational domain is the rectangle $\Omega=(0,4) \times(0,1)$. The following pre-shock and post-shock values of the flow variables are used

$$
\left[\begin{array}{l}
\rho_{L} \\
u_{L} \\
v_{L} \\
p_{L}
\end{array}\right]=\left[\begin{array}{c}
8.0 \\
8.25 \cos \left(30^{\circ}\right) \\
-8.25 \sin \left(30^{\circ}\right) \\
116.5
\end{array}\right], \quad\left[\begin{array}{l}
\rho_{R} \\
u_{R} \\
v_{R} \\
p_{R}
\end{array}\right]=\left[\begin{array}{l}
1.4 \\
0.0 \\
0.0 \\
1.0
\end{array}\right] .
$$

Initially, the post-shock values are prescribed in $\Omega_{L}=\left\{(x, y) \mid x<\frac{1}{6}+\frac{y}{\sqrt{3}}\right\}$ and the pre-shock values in $\Omega_{R}=\Omega \backslash \Omega_{L}$. The reflecting wall corresponds to $1 / 6 \leq x \leq 4$ and $y=0$. No boundary conditions are required along the line $x=4$. On the rest of the boundary, the post-shock conditions are prescribed for $x<\frac{1}{6}+\frac{1+20 t}{\sqrt{3}}$ and the pre-shock conditions elsewhere. The so-defined values along the top boundary describe the exact motion of the shock. 
(a) HLL- $P_{0}$

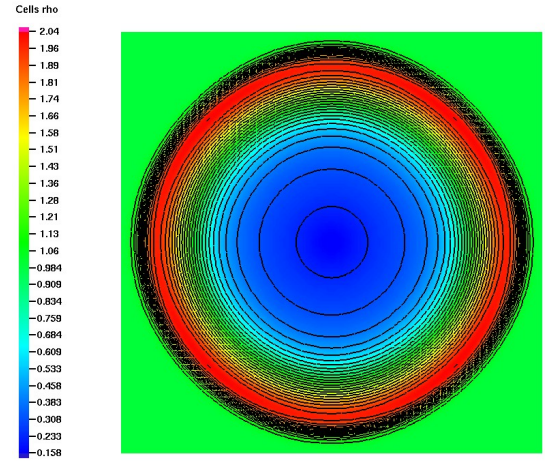

(b) HLL- $P_{1}$-VBC

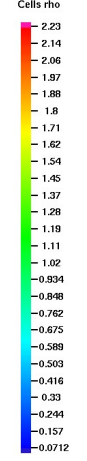

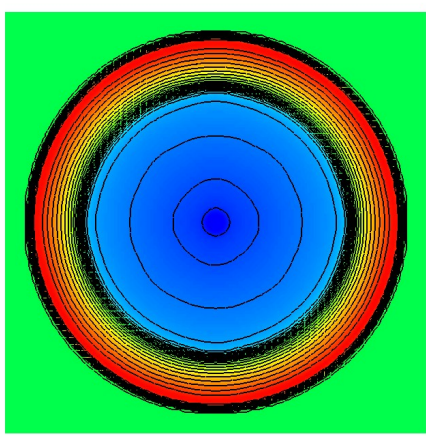

Figure 10: Radially symmetric Riemann problem: density at $t=0.13$.

(a) $x=0$ vs. $y=0$

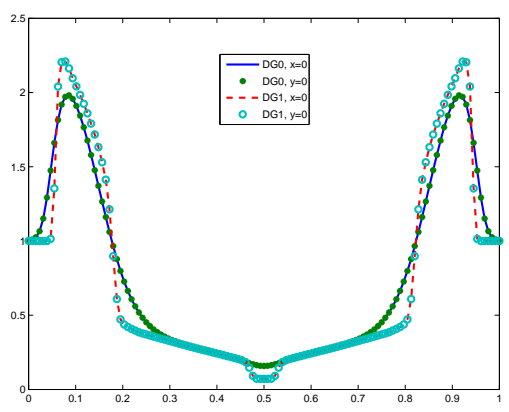

(b) $x=y$ vs. $x=-y$

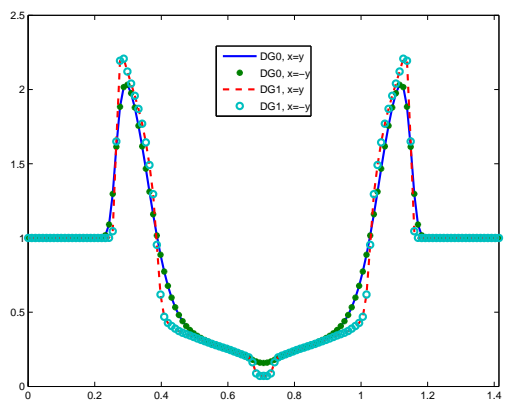

Figure 11: Radially symmetric Riemann problem: density cutlines at $t=0.13$.

The diagrams in Fig. 12 display snapshots of the density distribution at $t=0.2$. A uniform mesh of 65,536 rectangular elements and the time step $\Delta t=10^{-4}$ were employed in this test. The HLL- $P_{0}$ solution (Fig. 12a) exhibits strong numerical diffusion, which leads to a poor resolution of the interacting shock waves. The results obtained with the HLL- $P_{1}$ scheme reveal that the structure of the flow pattern near the triple point is more complex and calls for adaptive mesh refinement. It can be seen that the slope-limited $P_{1}$ solutions are free of 'staircase structures' and other artifacts observed by Woodward and Colella [46]. In this example, the optional control of the velocity and energy density (Fig. 12c,d) yields just a marginal improvement compared to segregated limiting of the conservative variables (Fig. 12b). 
(a) HLL- $P_{0}$

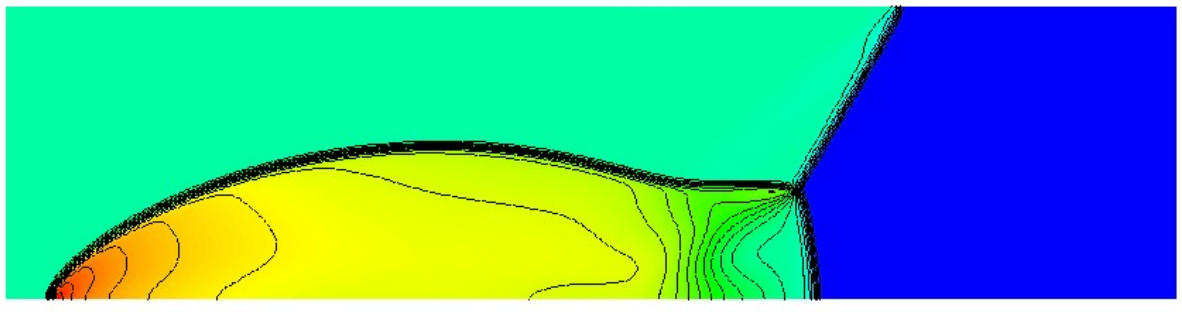

(b) HLL- $P_{1}$-VB

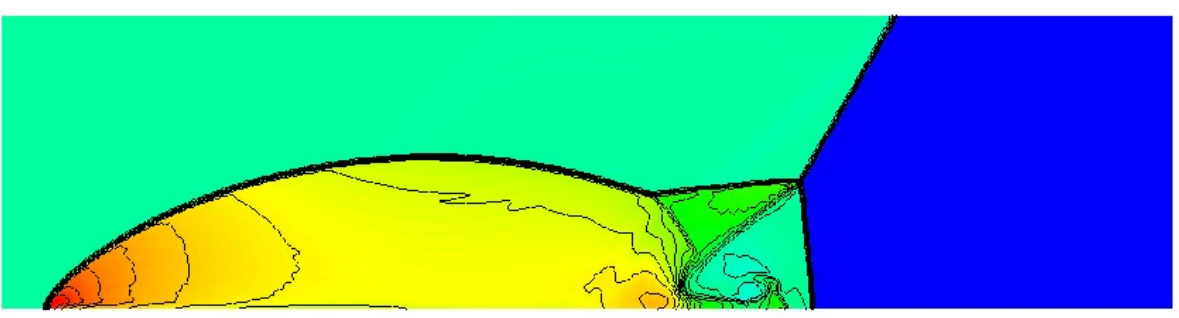

(c) HLL- $P_{1}$-VBC

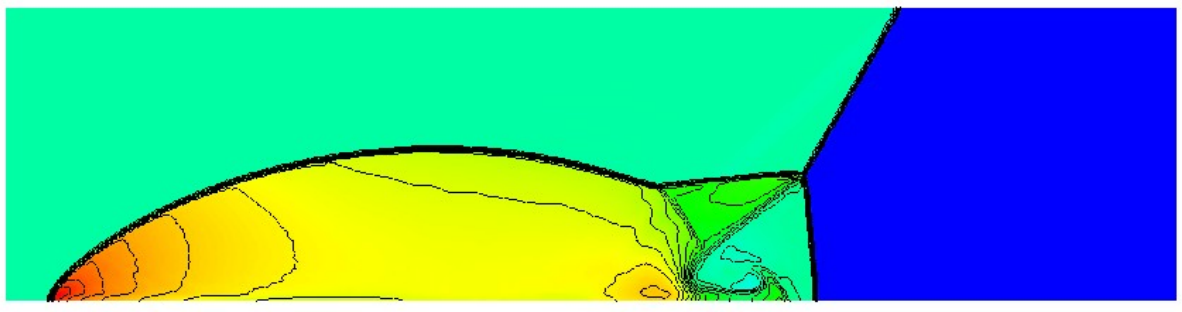

(d) HLL- $P_{2}$-VBC

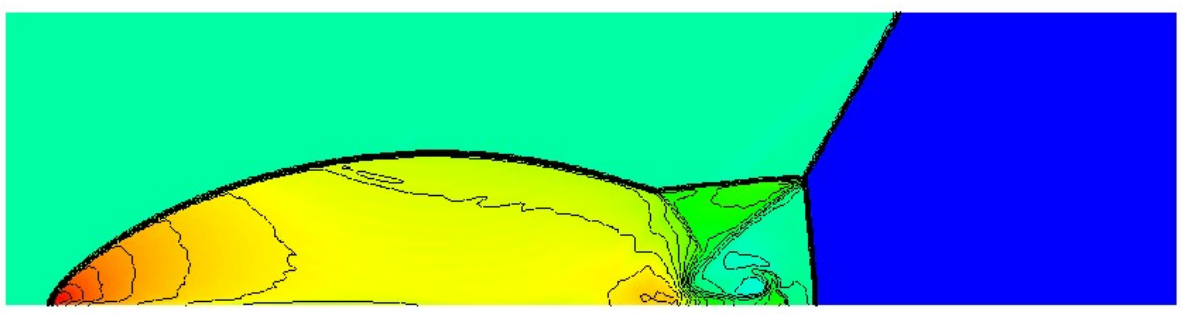

Figure 12: Double Mach reflection: density at $t=0.2$. 


\section{Conclusions}

The main outcome of the presented research is a new perspective on hierarchical slope limiting in explicit and implicit DG methods. The variational multiscale framework has motivated an iterative coupling of an implicit global problem for the cell mean values with small local problems for the derivatives of the DG solution. The developed iterative method enjoys unconditional stability, which makes it a robust tool for steady state computations. The replacement of slope limiting by solution of local inequality-constrained optimization problems was found to have a beneficial effect on convergence to steady-state solutions. Also, we proposed a new definition of bounds for boundary nodes, discussed the use of reconstruction techniques and introduced a compatible gradient limiting procedure for the Euler equations.

The above methodology provides a fairly general framework for the design of constrained DG methods. The presented algorithms produce promising results but there is still a lot of room for further research. In particular, a more careful numerical study of slope limiting and constrained minimization in implicit DG schemes is required. The presented iterative solver may be accelerated using a properly configured $p$-multigrid method [31]. Further work will also explore the possibility of enriching a continuous $P_{1} / Q_{1}$ Galerkin approximation with higher-order discontinuous basis functions. This approach seems to offer substantial efficiency gains compared to the pure DG method. The discrete maximum principle for the continuous coarse-scale components can be readily enforced using algebraic flux correction schemes [23].

\section{Acknowledgments}

This research was supported by the German Research Association (DFG) under grant KU 1530/6-1. The author would like to thank Dr. Vadym Aizinger (University Erlangen-Nuremberg) for inspiring discussions.

\section{References}

[1] V. Aizinger, A geometry independent slope limiter for the discontinuous Galerkin method. In: Notes on Numerical Fluid Mechanics and Multidisciplinary Design, Vol. 115 (2011) 207-217.

[2] T. Barth and D.C. Jespersen, The design and application of upwind schemes on unstructured meshes. AIAA Paper, 89-0366, 1989. 
[3] R. Biswas, K. Devine, and J. E. Flaherty, Parallel adaptive finite element methods for conservation laws. Appl. Numer. Math. 14 (1994) 255-284.

[4] M. Bittl and D. Kuzmin, An hp-adaptive flux-corrected transport algorithm for continuous finite elements. Computing, in press, DOI:10.1007/s00607-012-0223-y.

[5] P.B. Bochev, T.J.R. Hughes, and G. Scovazzi, A multiscale discontinuous Galerkin method. In I. Lirkov et al. (Eds.), Lecture Notes in Computer Science, Vol. 3743, pp. 84-93, Springer, 2006.

[6] P. Bochev, D. Ridzal, G. Scovazzi, and M. Shashkov, Constrainedoptimization based data transfer / A new perspective on flux correction. In: D. Kuzmin et al. (Eds.) Flux-Corrected Transport: Principles, Algorithms, and Applications, Springer, 2012, pp. 345-398.

[7] A. Burbeau, P. Sagaut, and C.-H. Bruneau, A problem-independent limiter for high-order Runge-Kutta discontinuous Galerkin methods. $J$. Comput. Phys. 169 (2001) 111-150.

[8] B. Cockburn, G.E. Karniadakis, and C.-W. Shu, The development of discontinuous Galerkin methods. In: B. Cockburn, G.E. Karniadakis, and C.-W. Shu (eds), Discontinuous Galerkin Methods. Theory, Computation and Applications, LNCSE 11 (2000), Springer, New York, 3-50.

[9] B. Cockburn and C.-W. Shu, Runge-Kutta discontinuous Galerkin methods for convection-dominated problems. J. Sci. Comput. 16 (2001) 173-261.

[10] B. Cockburn and C.-W. Shu, The Runge-Kutta discontinuous Galerkin method for conservation laws V. Multidimensional Systems. J. Comput. Phys. 141 (1998) 199-224.

[11] V. Dolejší and M. Feistauer, On the discontinuous Galerkin method for the numerical solution of compressible high-speed flow. In: Numerical Mathematics and Advanced Applications, ENUMATH 2001, F. Brezzi, A. Buffa, S. Corsaro, and A. Murli (eds.), Springer-Verlag, Berlin, 2003, pp. 65-83. 
[12] M. Dumbser, Arbitrary high order $P_{n} P_{m}$ schemes on unstructured meshes for the compressible NavierStokes equations. Computers $\mathcal{E}$ Fluids 39 (2010) 60-76.

[13] M. Dumbser, D.S. Balsara, E.F. Toro, and C.D. Munz, A unified framework for the construction of one-step finite volume and discontinuous Galerkin schemes on unstructured meshes. J. Comput. Phys. 227 (2008) 8209-8253.

[14] M. Feistauer and V. Kučera, On a robust discontinuous Galerkin technique for the solution of compressible flow. J. Comp. Phys. 224 (2007) 208-231.

[15] J.E. Flaherty, L. Krivodonova, J.-F. Remacle, and M.S. Shephard, Aspects of discontinuous Galerkin methods for hyperbolic conservation laws. Finite Elements in Analysis and Design 38 (2002) 889-908.

[16] S. Gottlieb and C.-W. Shu, Total Variation Diminishing Runge-Kutta schemes. Math. Comp. 67 (1998) 73-85.

[17] S. Gottlieb, C.-W. Shu, and E. Tadmor, Strong stability-preserving highorder time discretization methods. SIAM Review 43 (2001) 89-112.

[18] A. Harten, P. D. Lax and B. van Leer, On upstream differencing and Godunov type schemes for hyperbolic conservation laws, SIAM Rev. 25 (1983) 33-61.

[19] H. Hoteit, Ph. Ackerer, R. Mosé, J. Erhel, and B. Philippe, New twodimensional slope limiters for discontinuous Galerkin methods on arbitrary meshes. Int. J. Numer. Meth. Engrg. 61 (2004) 2566-2593.

[20] T.J.R. Hughes, G. Scovazzi, P.B. Bochev, and A. Buffa, A multiscale discontinuous Galerkin method with the computational structure of a continuous Galerkin method, Comp. Meth. Appl. Mech. Engrg. 195 (2006) 2761-2787.

[21] L. Krivodonova, Limiters for high-order discontinuous Galerkin methods. J. Comput. Phys. 226 (2007) 879-896. 
[22] L. Krivodonova, J. Xin, J.-F. Remacle, N. Chevaugeon, and J.E. Flaherty, Shock detection and limiting with discontinuous Galerkin methods for hyperbolic conservation laws. Appl. Numer. Math. 48 (2004) 323-338.

[23] D. Kuzmin, Explicit and implicit FEM-FCT algorithms with flux linearization. J. Comput. Phys. 228 (2009) 2517-2534.

[24] D. Kuzmin, A vertex-based hierarchical slope limiter for p-adaptive discontinuous Galerkin methods. J. Comput. Appl. Math. 233 (2010) 30773085 .

[25] D. Kuzmin, Slope limiting for discontinuous Galerkin approximations with a possibly non-orthogonal Taylor basis. Int. J. Numer. Methods Fluids, in press, DOI: 10.1002/fld.3707.

[26] D. Kuzmin, M. Möller and M. Gurris, Algebraic flux correction II. Compressible flow problems, In: D. Kuzmin, R. Löhner and S. Turek (Eds), Flux-Corrected Transport: Principles, Algorithms, and Applications, Springer, 2nd edition, 2012, pp. 193-238.

[27] D. Kuzmin, M. Möller, J.N. Shadid and M. Shashkov: Failsafe flux limiting and constrained data projections for equations of gas dynamics. J. Comput. Phys. 229 (2010) 8766-8779.

[28] D. Kuzmin and F. Schieweck, A parameter-free smoothness indicator for derivatives of finite element solutions. CEJM Topical Issue Numerical Methods for Large Scale Scientific Computing, 2013, accepted.

[29] R. J. LeVeque, Simplified multi-dimensional flux limiting methods. $\mathrm{Nu}$ merical Methods for Fluid Dynamics IV (1993) 175-190.

[30] R.J. LeVeque, High-resolution conservative algorithms for advection in incompressible flow. SIAM J. Numer. Anal. 33 (1996) 627-665.

[31] H. Luo, J.D. Baum, and R. Löhner, Fast p-multigrid discontinuous Galerkin method for compressible flows at all speeds. AIAA Journal 46 (2008) 635-652.

[32] H. Luo, J.D. Baum, and R. Löhner, A discontinuous Galerkin method based on a Taylor basis for the compressible flows on arbitrary grids. J. Comput. Phys. 227 (2008) 8875-8893. 
[33] H. Luo, H. Segawa, and M.R. Visbal, An implicit discontinuous Galerkin method for the unsteady compressible Navier-Stokes equations. Computers \& Fluids, 53 (2012) 133-144.

[34] H. Luo, Y. Xia, S. Li, R. Nourgaliev, and C. Cai, A Hermite WENO reconstruction-based discontinuous Galerkin method for the Euler equations on tetrahedral grids. J. Comput. Phys. 231 (2012) 5489-5503.

[35] K. Michalak and C. Ollivier-Gooch, Limiters for unstructured higherorder accurate solutions of the Euler equations. In: Proceedings of the AIAA Forty-Sixth Aerospace Sciences Meeting, 2008.

[36] C. Michoski, C. Mirabito, C. Dawson, E.J Kubatko, D. Wirasaet, and J.J. Westerink, Adaptive hierarchic transformations for dynamically $p$ enriched slope-limiting over discontinuous Galerkin systems of generalized equations. Submitted to J. Comput. Phys. (2010).

[37] W.J. Rider and D.B. Kothe, Constrained minimization for monotonic reconstruction. Proceedings of the Thirteenth AIAA Computational Fluid Dynamics Conference, 1997, pp. 955-964.

[38] G. Sod, A survey of several finite difference methods for systems of nonlinear hyperbolic conservation laws. J. Comput. Phys. 27 (1978) 131.

[39] E. F. Toro, Riemann Solvers and Numerical Methods for Fluid Dynamics. Springer, 1999.

[40] J.-Y. Trépanier, M. Reggio and D. Ait-Ali-Yahia, An implicit flux-difference splitting method for solving the Euler equations on adaptive triangular grids. Int. J. Num. Meth. Heat Fluid Flow 3 (1993) 63-77.

[41] S. Tu and S. Aliabadi, A slope limiting procedure in discontinuous Galerkin finite element method for gasdynamics applications. Int. J. Numer. Anal. Model. 2 (2005) 163-178.

[42] M. Yang and Z.J. Wang, A parameter-free generalized moment limiter for high-order methods on unstructured grids, AIAA-2009-605.

[43] W.B. VanderHeyden and B.A. Kashiwa, Compatible fluxes for van Leer advection, J. Comput. Phys. 146 (1998) 1-28. 
[44] V. Venkatakrishnan, Convergence to steady state solutions of the Euler equations on unstructured grids with limiters. J. Comput. Phys. 118 (1995) 120-130.

[45] F. Vilar, Cell-centered discontinuous Galerkin discretization for twodimensional Lagrangian hydrodynamics. Computers \&3 Fluids 64 (2012) 64-73.

[46] P. R. Woodward and P. Colella, The numerical simulation of twodimensional fluid flow with strong shocks. J. Comput. Phys. 54 (1984) $115-173$. 